\title{
Phase trapping in multistep spin crossover compound
}

Fabian Fürmeyer, Luca M. Carrella, Vadim Ksenofontov, Angela Möller and Eva Rentschler* Department of Chemistry

Johannes Gutenberg University Mainz

Duesbergweg 10 - 14, 55128 Mainz

E-mail: rentschler@uni-mainz.de 


\section{Table of content}

NMR spectra

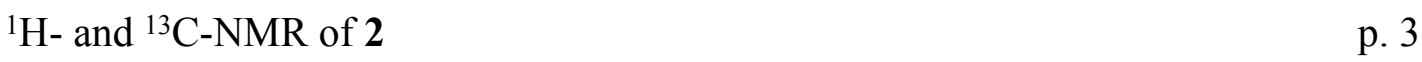

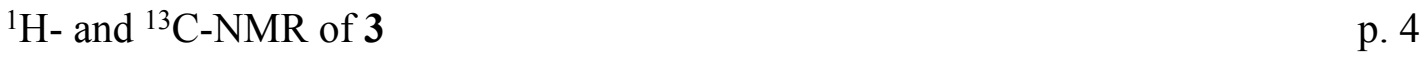

${ }^{1} \mathrm{H}$ - and ${ }^{13} \mathrm{C}-\mathrm{NMR}$ of $\mathbf{L}^{\mathbf{1}}$ p. 5

${ }^{1} \mathrm{H}-$ and ${ }^{13} \mathrm{C}-\mathrm{NMR}$ of $\mathbf{L}^{2} \quad$ p. 6

$\begin{array}{ll}\text { IR spectra of dried } \mathbf{C 1}-\mathbf{C 6} & \text { p. } 7\end{array}$

X-ray diffraction measurements

$\begin{array}{ll}\text { Packing and asymmetric unit of } \mathbf{C 1} & \text { p. } 10\end{array}$

$\begin{array}{ll}\text { Selected bond lengths and angles for } \mathbf{C 1} & \text { p. } 11\end{array}$

Representation of the 1D chain and packing along the 1D chains of $\mathbf{C 4}-\mathbf{C 6} \quad$ p. 12

Molecular structure and asymmetric unit of $\mathbf{C 4}$ at $240 \mathrm{~K} \quad$ p. 13

Molecular structure and asymmetric unit of $\mathbf{C 4}$ at $120 \mathrm{~K} \quad$ p. 13

Molecular structure and asymmetric unit of $\mathbf{C 5}$ at $250 \mathrm{~K} \quad$ p. 14

Molecular structure, asymmetric unit and packing of C5 at $160 \mathrm{~K}$ (slow cooling) p. 14

Molecular structure and asymmetric unit of $\mathbf{C 5}$ at $160 \mathrm{~K}$ (quenching) p. 16

$\begin{array}{ll}\text { Molecular structure and asymmetric unit of } \mathbf{C 5} \text { at } 100 \mathrm{~K} & \text { p. } 16\end{array}$

$\begin{array}{ll}\text { Molecular structure and asymmetric unit of } \mathbf{C 6} \text { at } 100 \mathrm{~K} & \text { p. } 17\end{array}$

$\begin{array}{ll}\text { Crystallographic parameters for all structures of } \mathbf{C 1}-\mathbf{C 6} & \text { p. } 18\end{array}$

Magnetic data

$\begin{array}{ll}\text { Freshly prepared and dried samples of } \mathbf{C 1}-\mathbf{C 3} & \text { p. } 19\end{array}$

$\begin{array}{ll}\text { Freshly prepared and dried samples of } \mathbf{C 4} \text { and } \mathbf{C 5} & \text { p. } 19\end{array}$

$\begin{array}{ll}\text { C5 with a cooling rate of } 10 \mathrm{~K} / \mathrm{min} & \text { p. } 20\end{array}$ 
NMR spectroscopy

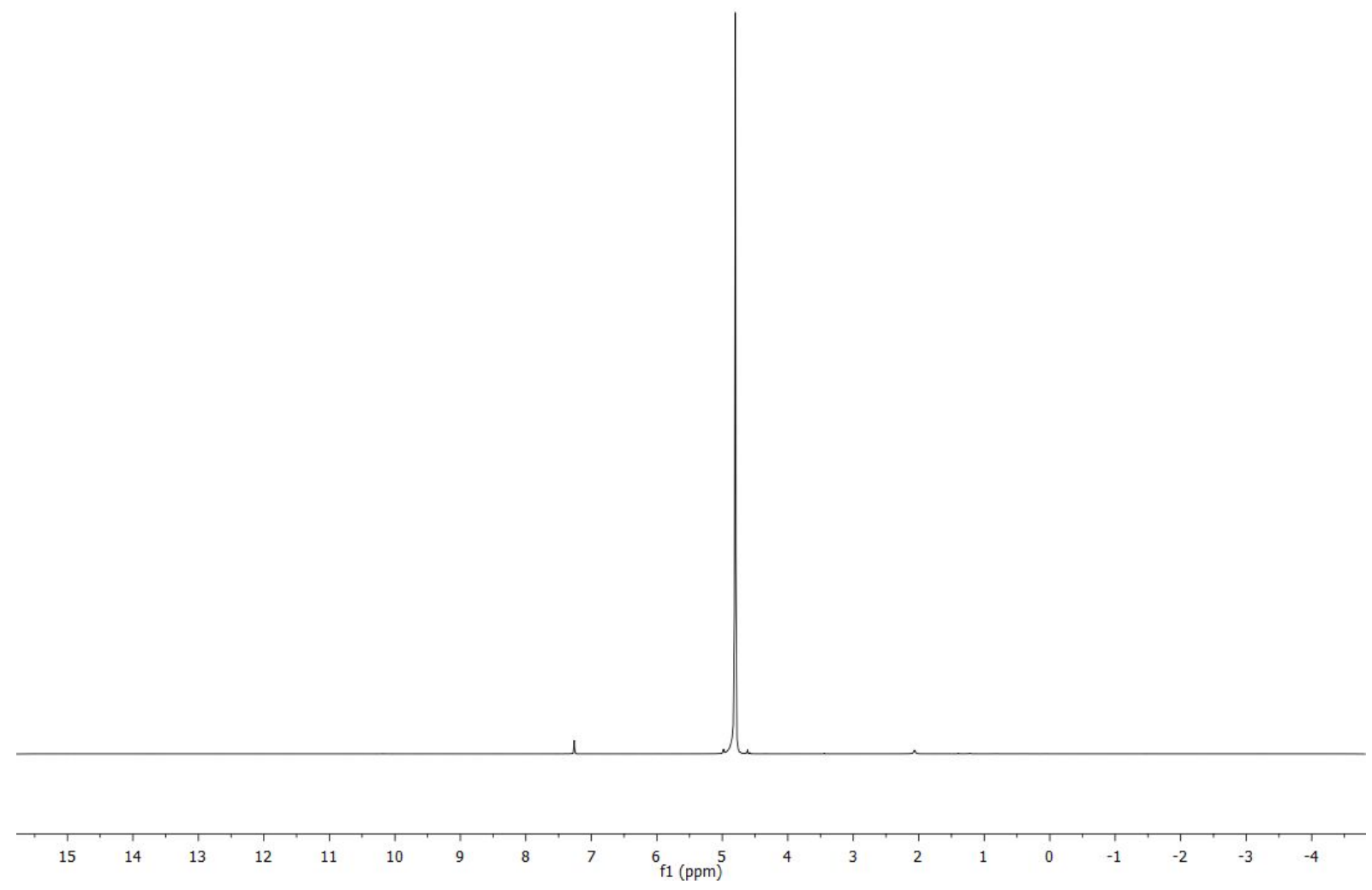

Figure S1: ${ }^{1} \mathrm{H}$-NMR of 2,5-bis(azidomethyl)-1,3,4-thiadiazole (2).

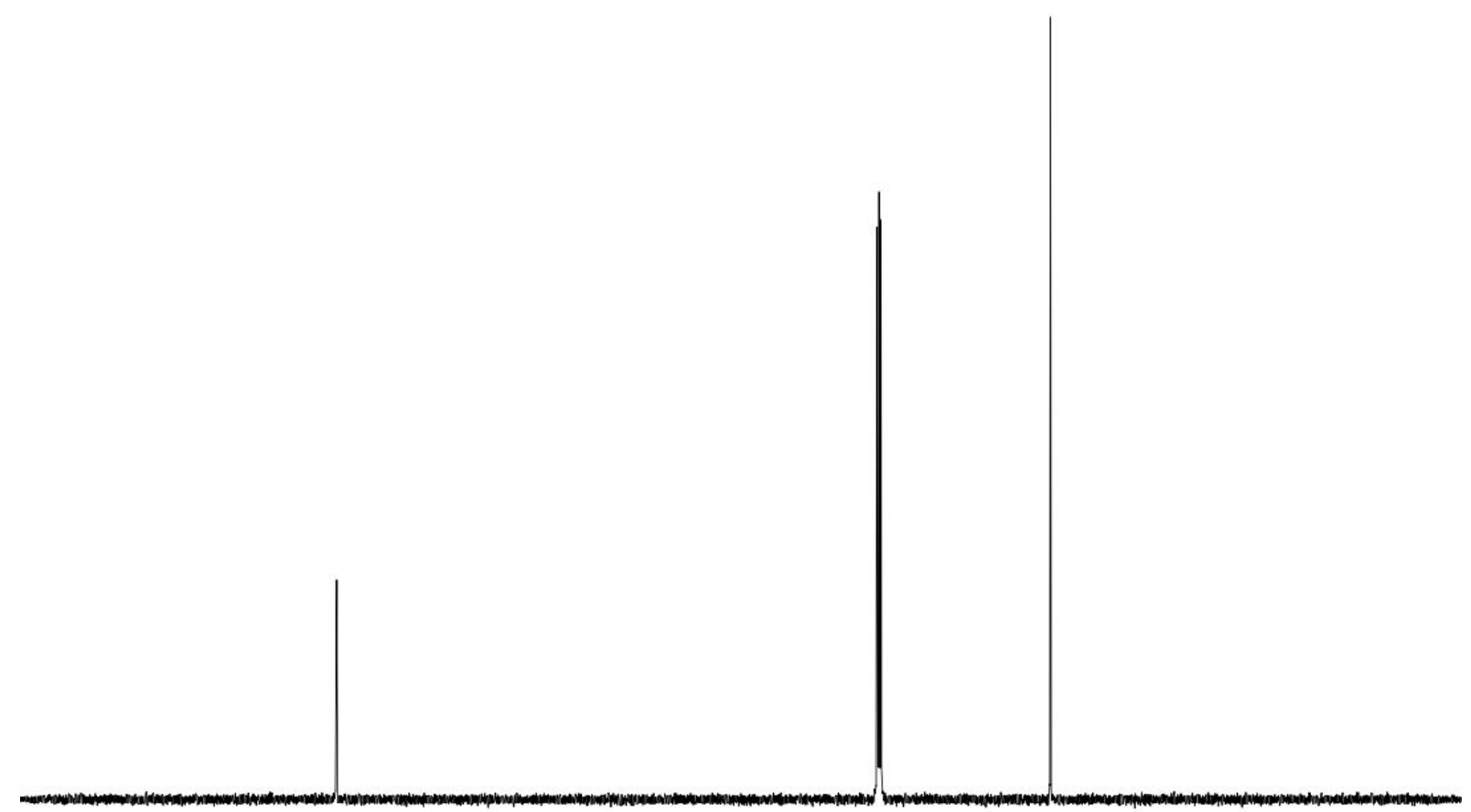

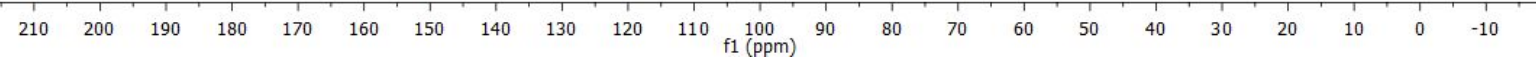

Figure S2: ${ }^{13} \mathrm{C}-\mathrm{NMR} 2,5$-bis(azidomethyl)-1,3,4-thiadiazole (2). 

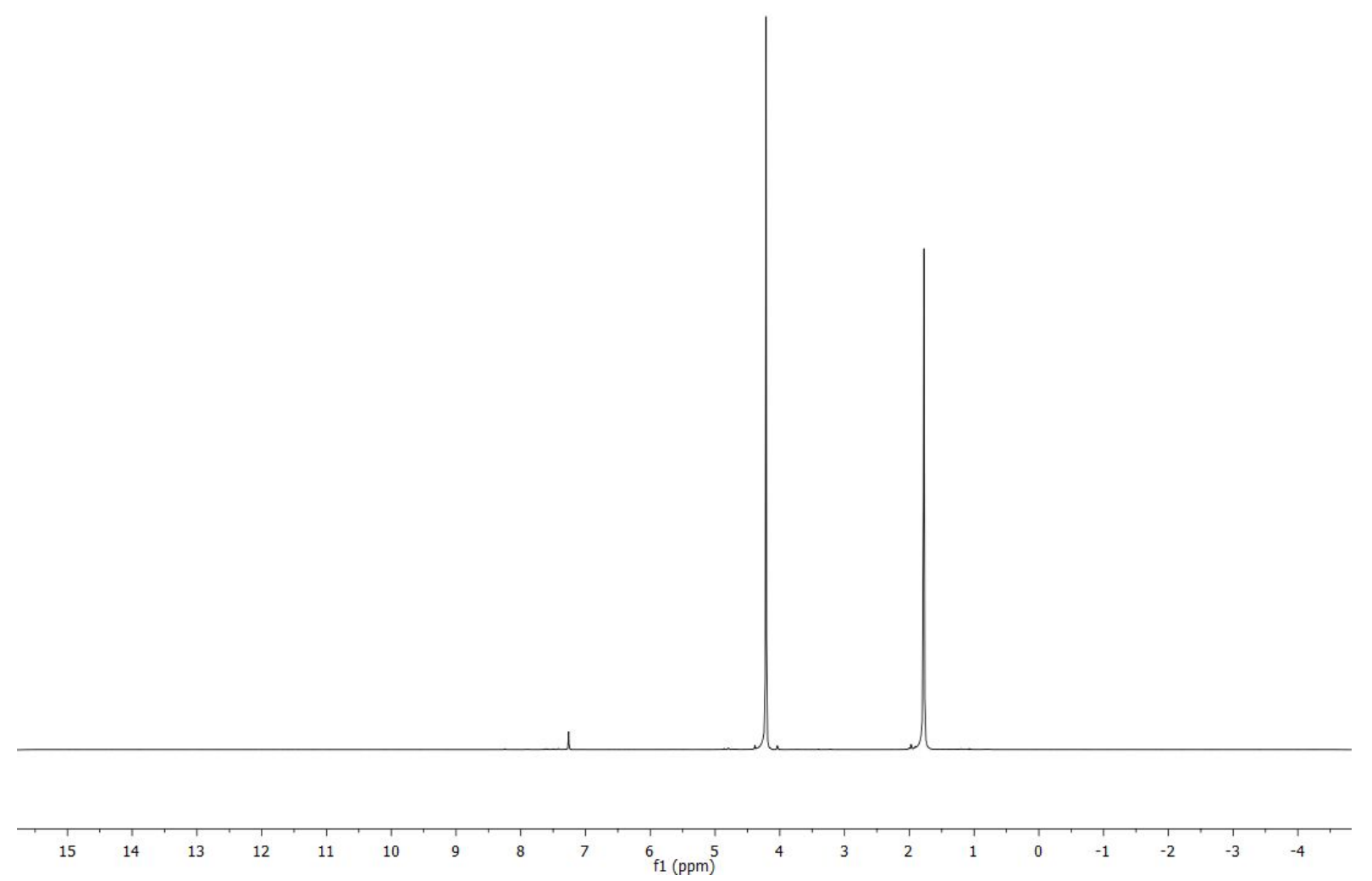

Figure S3: ${ }^{1} \mathrm{H}-\mathrm{NMR}$ of 2,5-bis(aminomethyl)-1,3,4-thiadiazole (3).

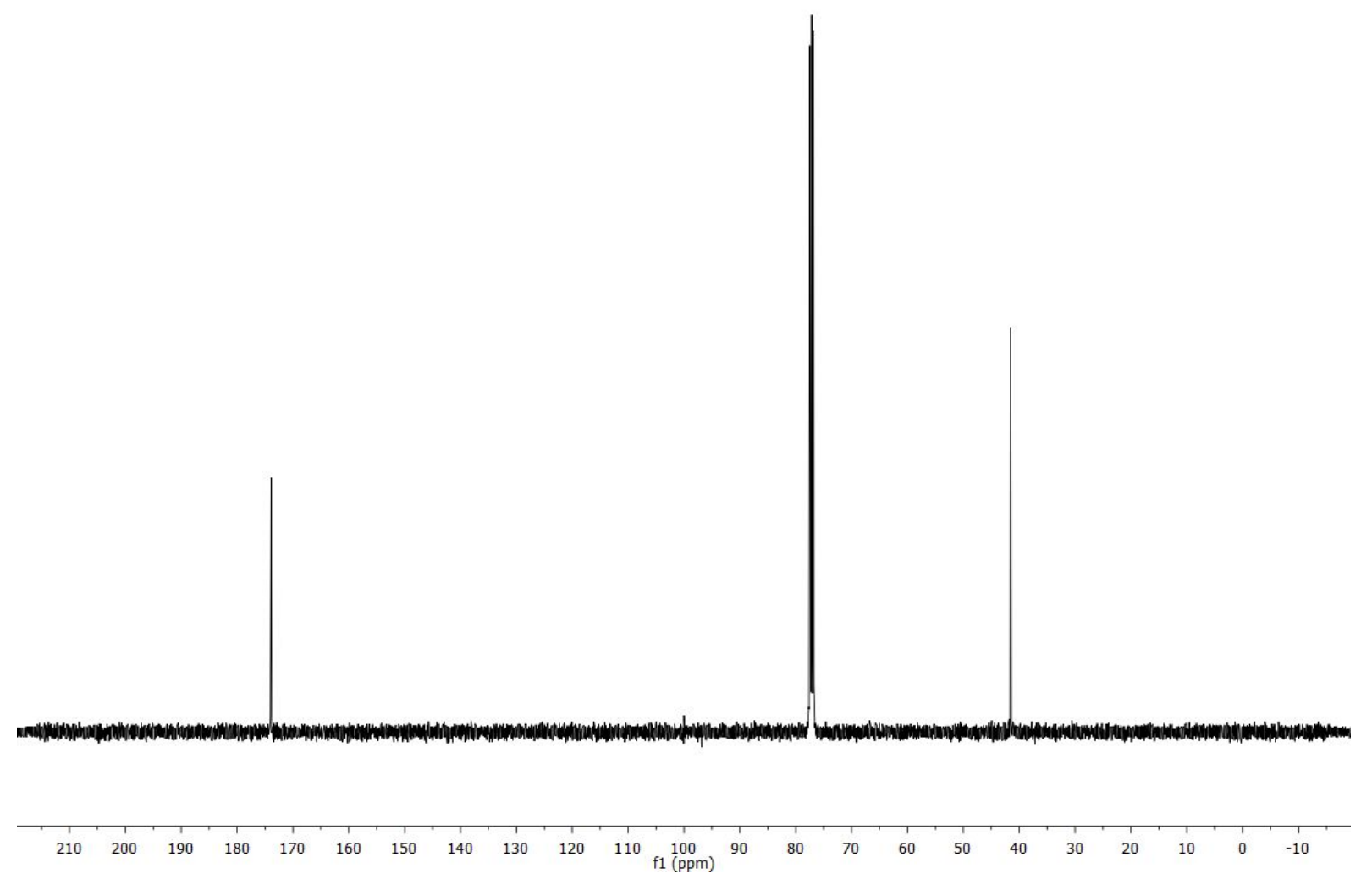

Figure S4: ${ }^{13} \mathrm{C}-\mathrm{NMR}$ of 2,5-bis(aminomethyl)-1,3,4-thiadiazole (3). 

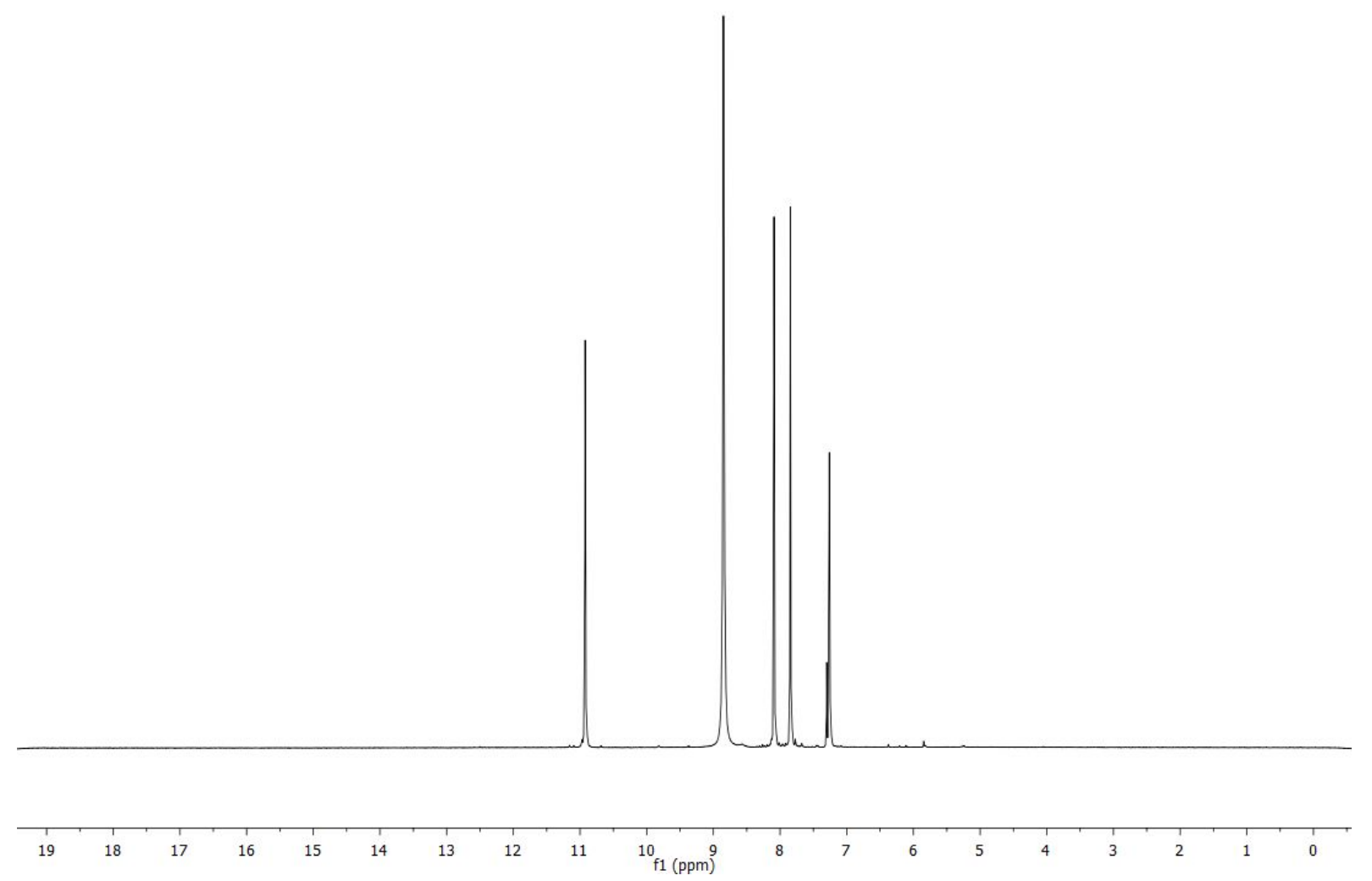

Figure S5: ${ }^{1} \mathrm{H}-\mathrm{NMR}$ of 2,5-Bis $\left\{[(1 \mathrm{H}\right.$-imidazol-2-ylmethyl)-amino $]$-methyl $\}$-1,3,4-thiadiazole $\left(\mathbf{L}^{1}\right)$.

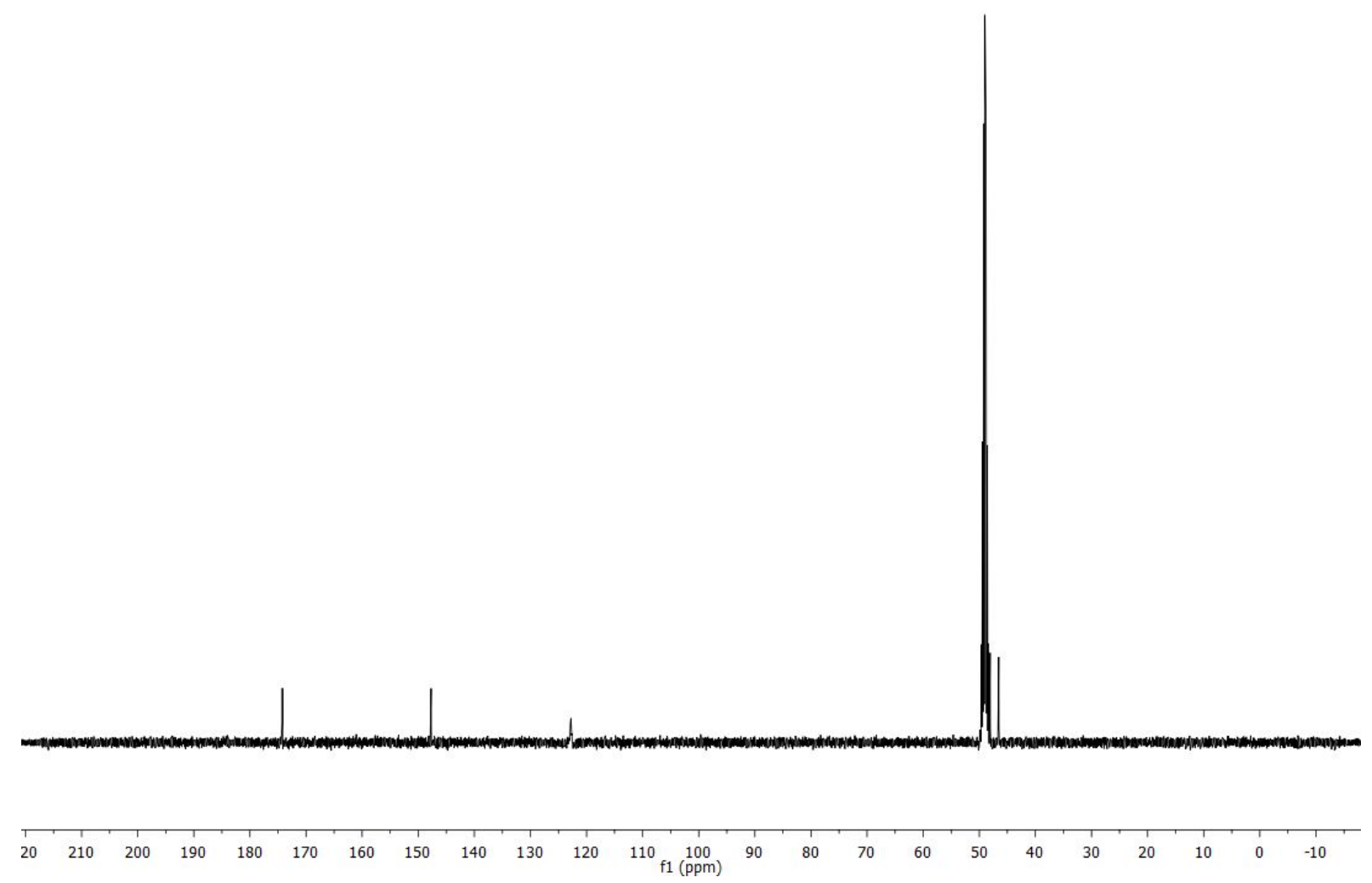

Figure S6: ${ }^{13} \mathrm{C}-\mathrm{NMR}$ of 2,5-Bis $\left\{[(1 \mathrm{H}\right.$-imidazol-2-ylmethyl)-amino $]$-methyl $\}$-1,3,4-thiadiazole $\left(\mathbf{L}^{\mathbf{1}}\right)$. 


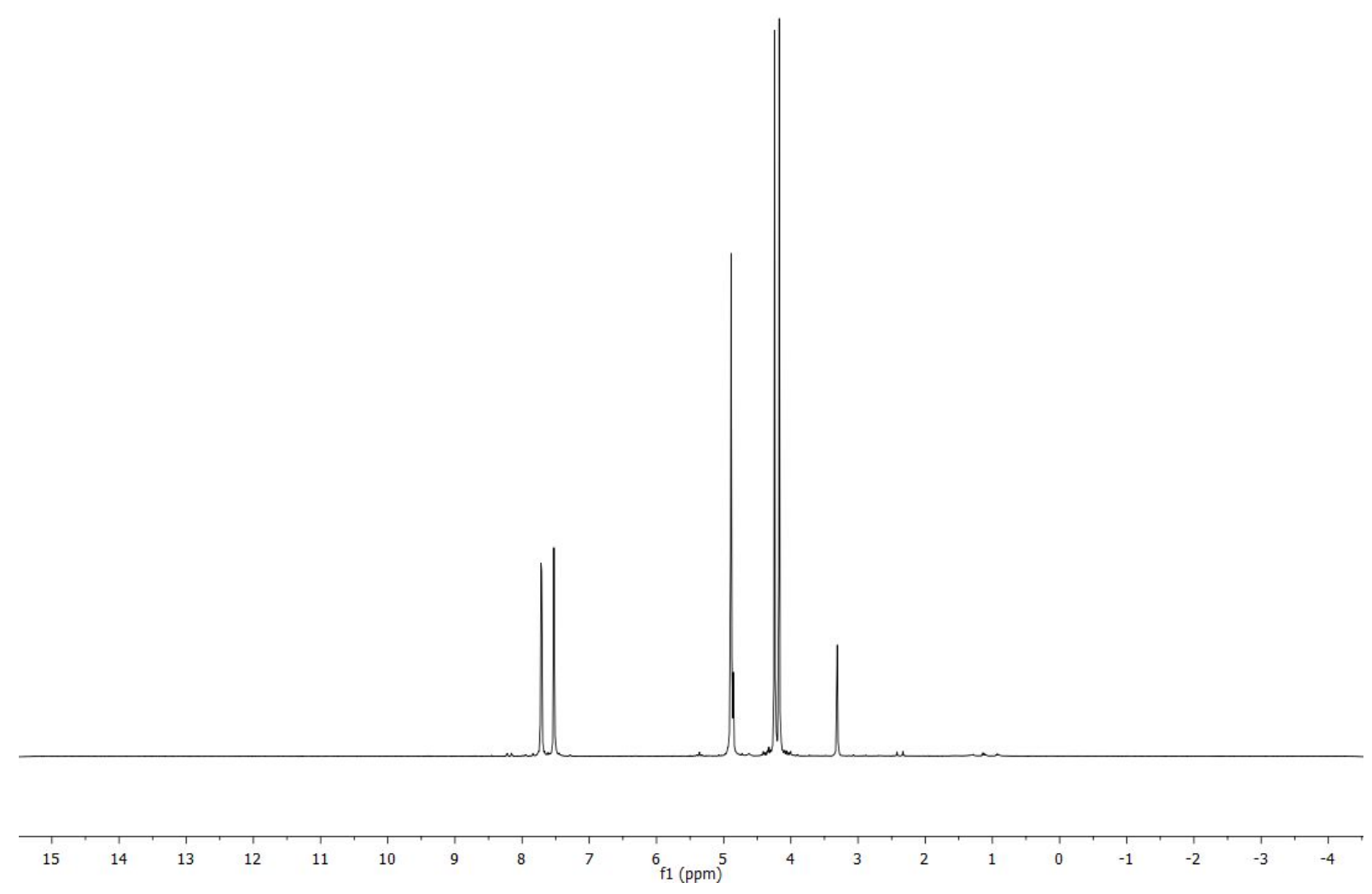

Figure S7: ${ }^{1} \mathrm{H}-\mathrm{NMR}$ of 2,5-Bis $\{[$ (thiazol-2-ylmethyl)-amino $]-m e t h y l\}-1,3,4$-thiadiazole $\left(\mathbf{L}^{2}\right)$.

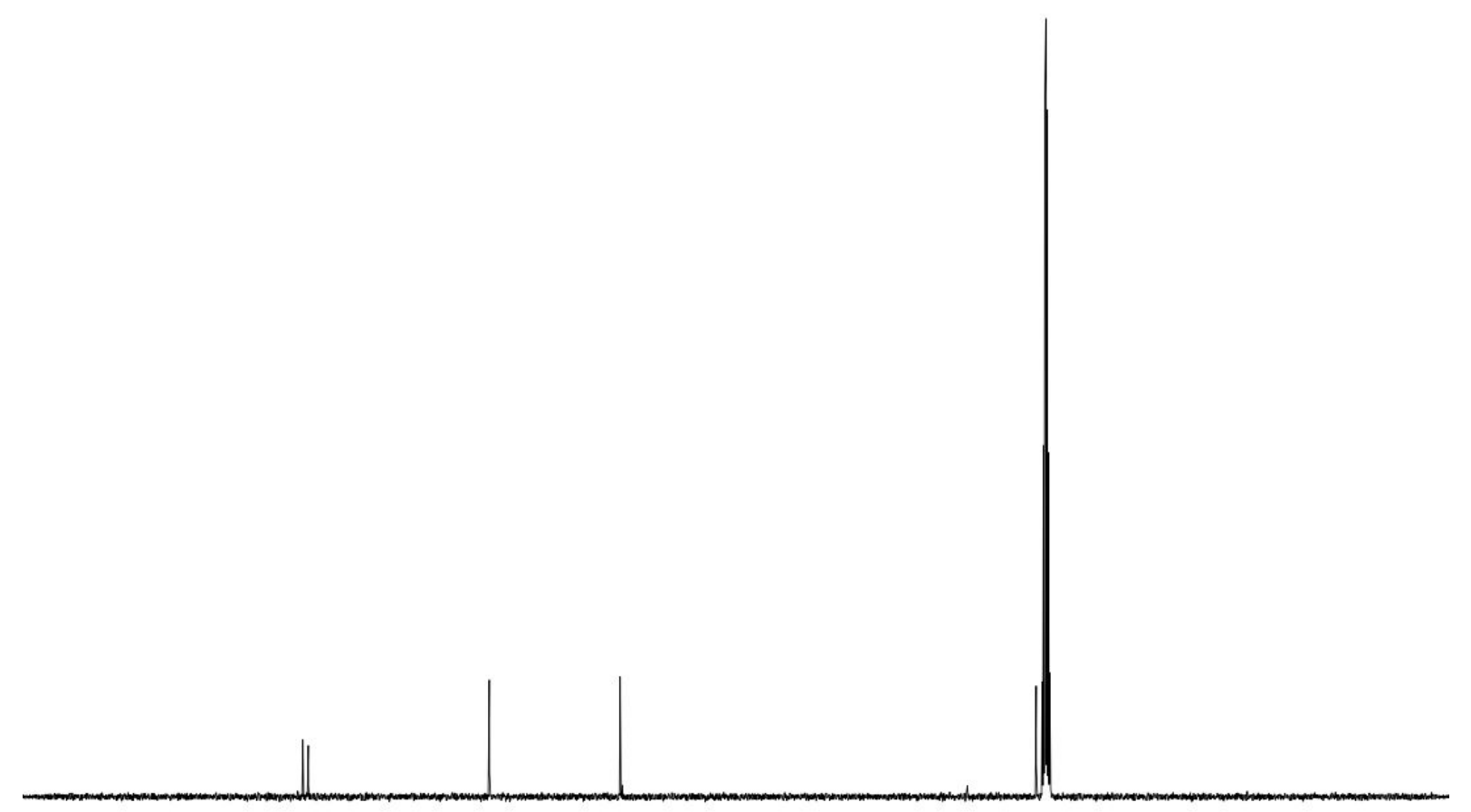

$\begin{array}{lllllllllllllllllllllllllllllll}220 & 210 & 200 & 190 & 180 & 170 & 160 & 150 & 140 & 130 & 120 & 110 & 100 & 90 & 80 & 70 & 60 & 50 & 40 & 30 & 20 & 10 & 0 & -10 & \end{array}$

Figure S8: ${ }^{13} \mathrm{C}-\mathrm{NMR}$ of 2,5-Bis $\left\{[(\right.$ thiazol-2-ylmethyl)-amino $]$-methyl $\}-1,3,4$-thiadiazole $\left(\mathbf{L}^{2}\right)$. 


\section{IR spectroscopy}

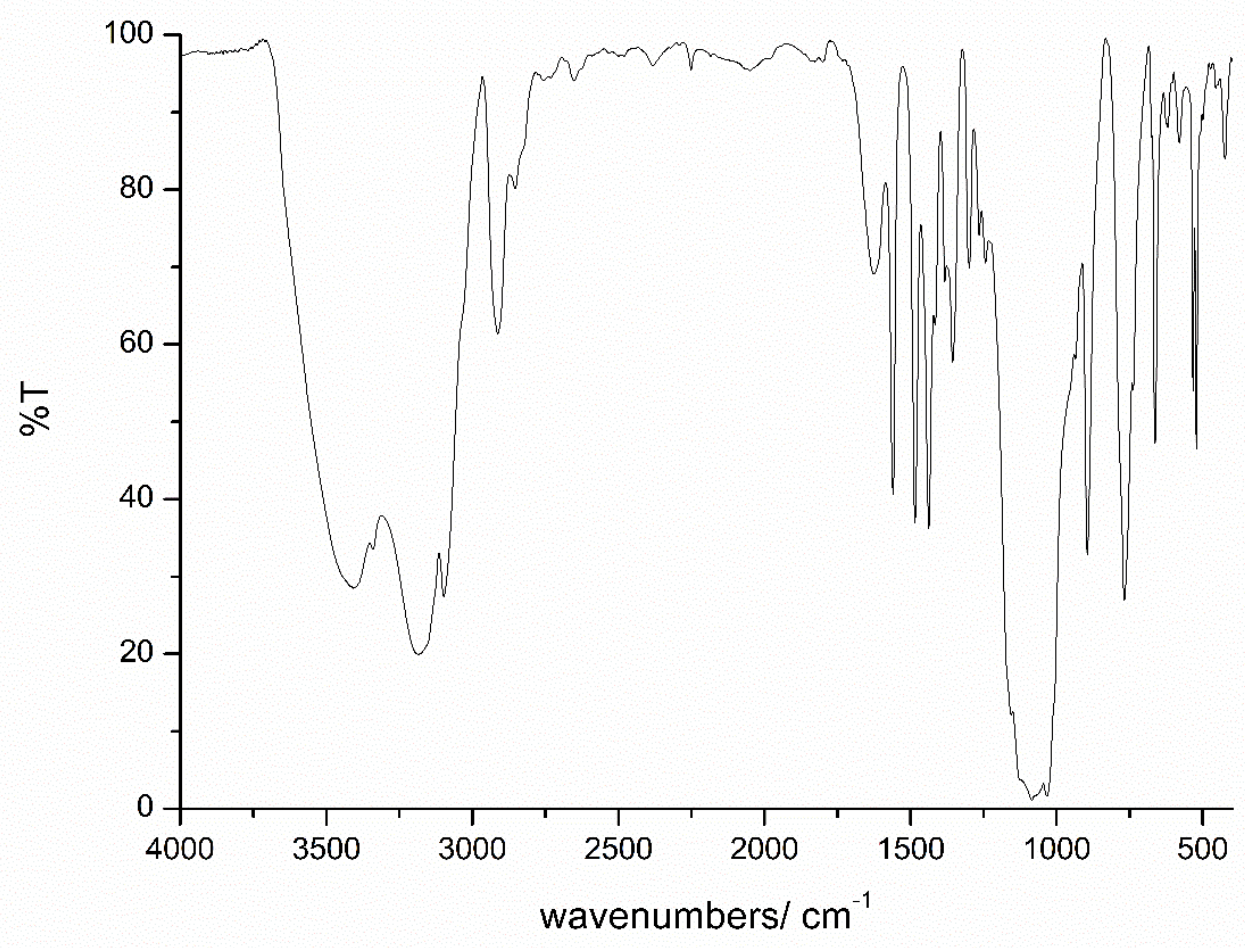

Figure S9: IR spectrum of dried $\left[\mathrm{Fe}_{2}{ }_{2}\left(\mu_{2}-\mathrm{L}^{1}\right)_{2}\right]\left(\mathrm{BF}_{4}\right)_{4}(\mathbf{C 1})$.

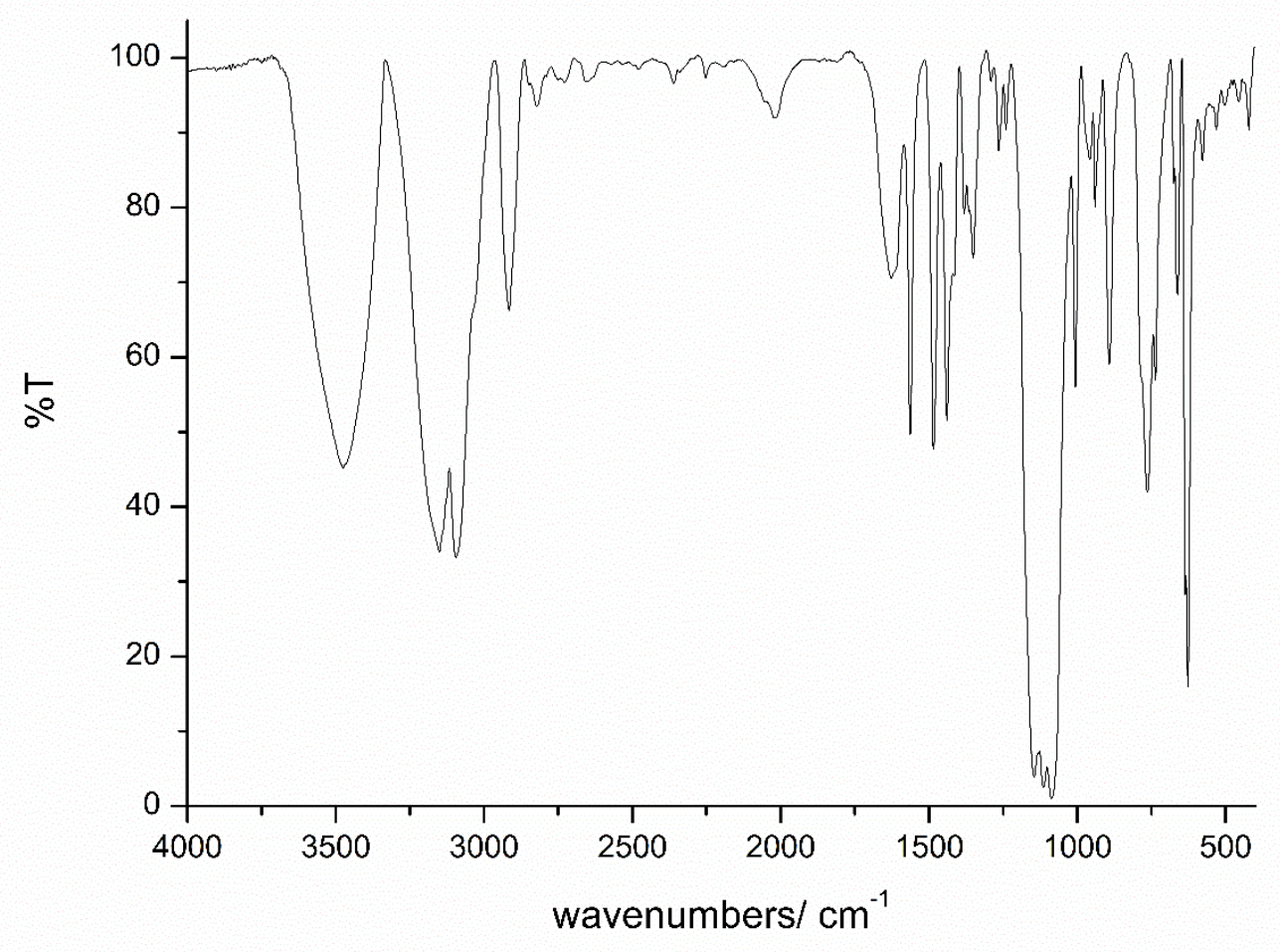

Figure S10: IR spectrum of dried $\left[\mathrm{Fe}_{2}{ }_{2}\left(\mu_{2}-\mathrm{L}^{1}\right)_{2}\right]\left(\mathrm{ClO}_{4}\right)_{4}(\mathbf{C} 2)$. 


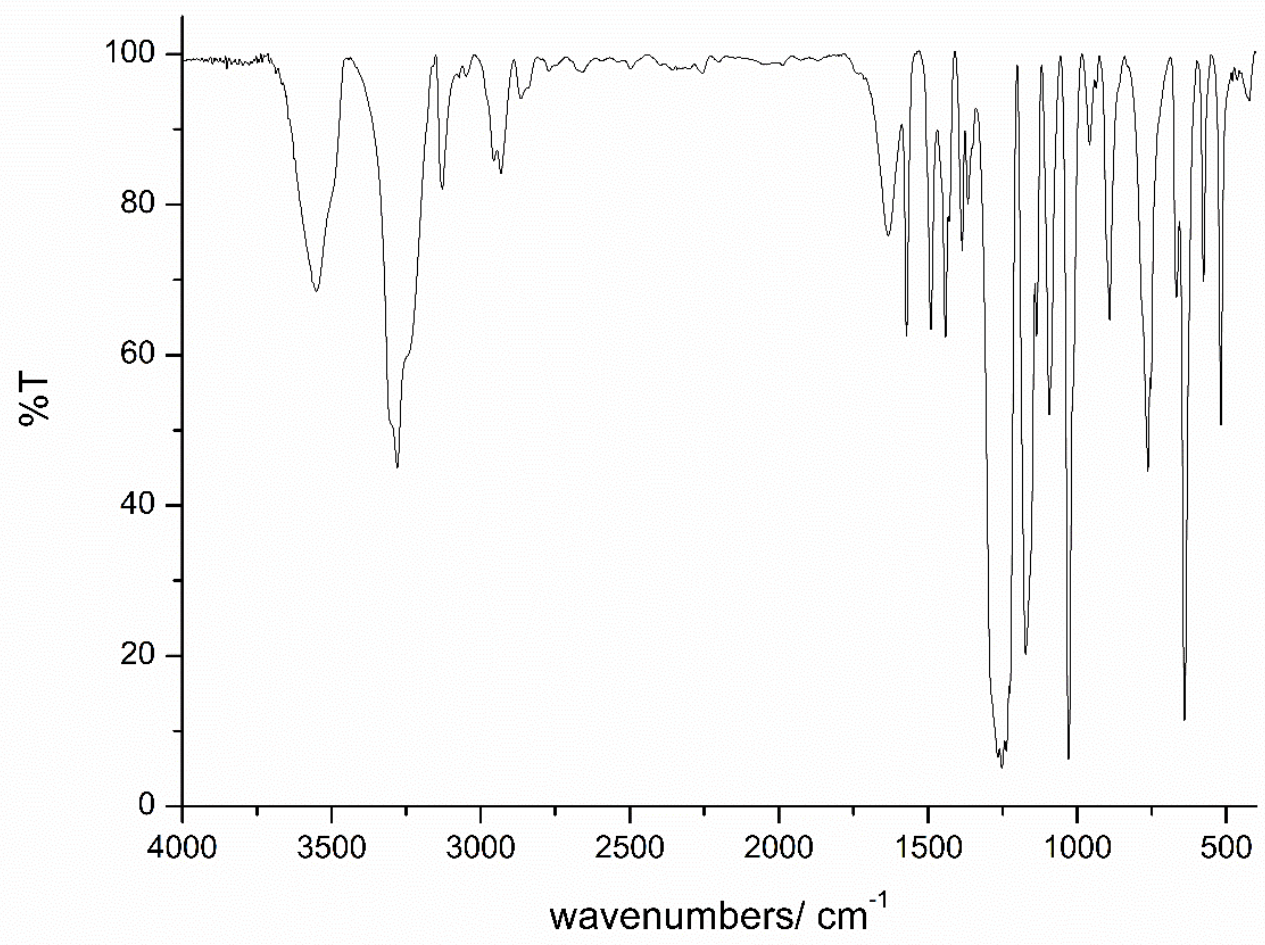

Figure S11: IR spectrum of dried $\left[\mathrm{Fe}^{\mathrm{II}}{ }_{2}\left(\mu_{2}-\mathrm{L}^{1}\right)_{2}\right]\left(\mathrm{F}_{3} \mathrm{CSO}_{3}\right)_{4}(\mathbf{C} 3)$.

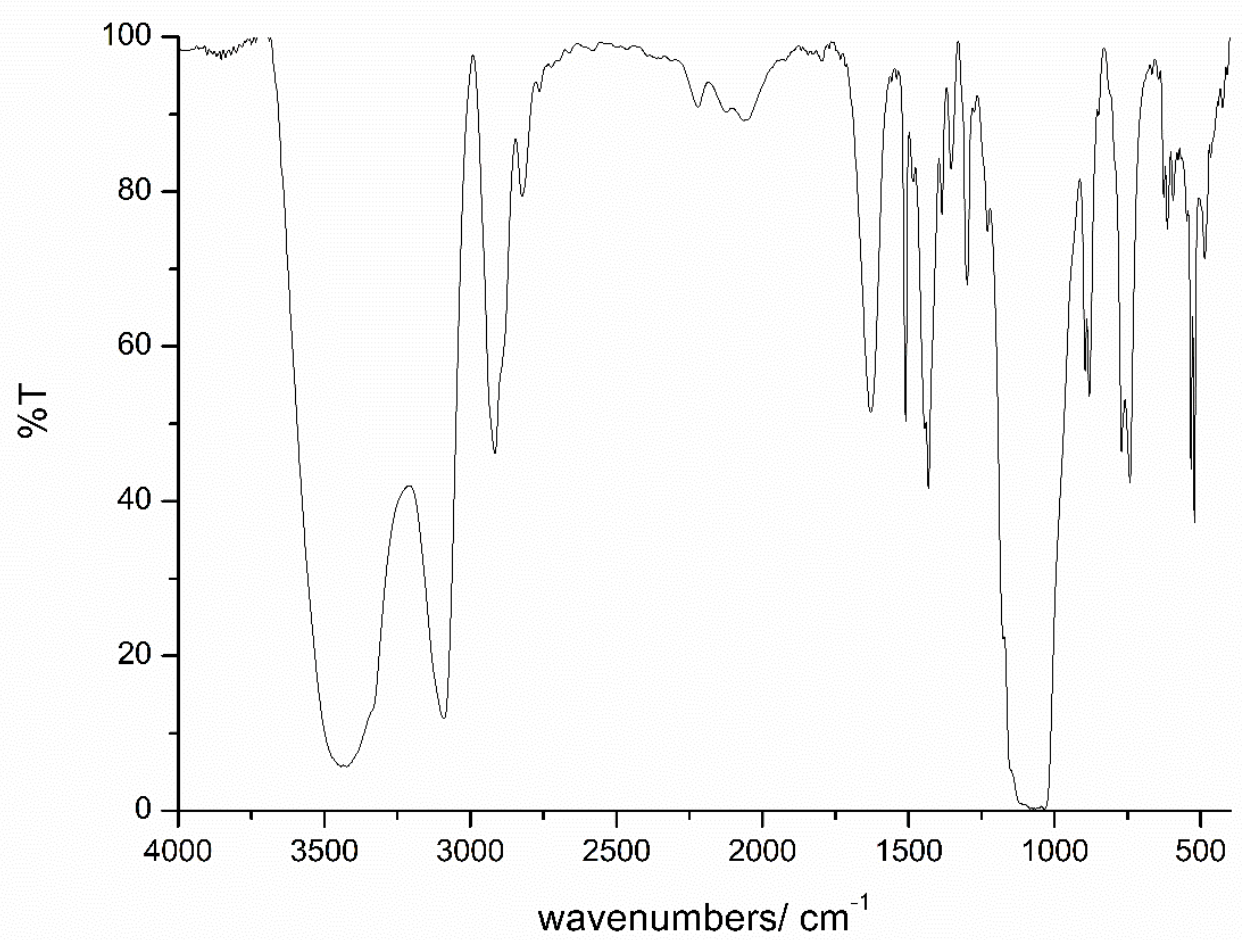

Figure S12: IR spectrum of dried $\left[\mathrm{Fe}_{2}{ }_{2}\left(\mu_{2}-\mathrm{L}^{2}\right)_{2}\right]\left(\mathrm{BF}_{4}\right)_{4}(\mathbf{C 4})$. 


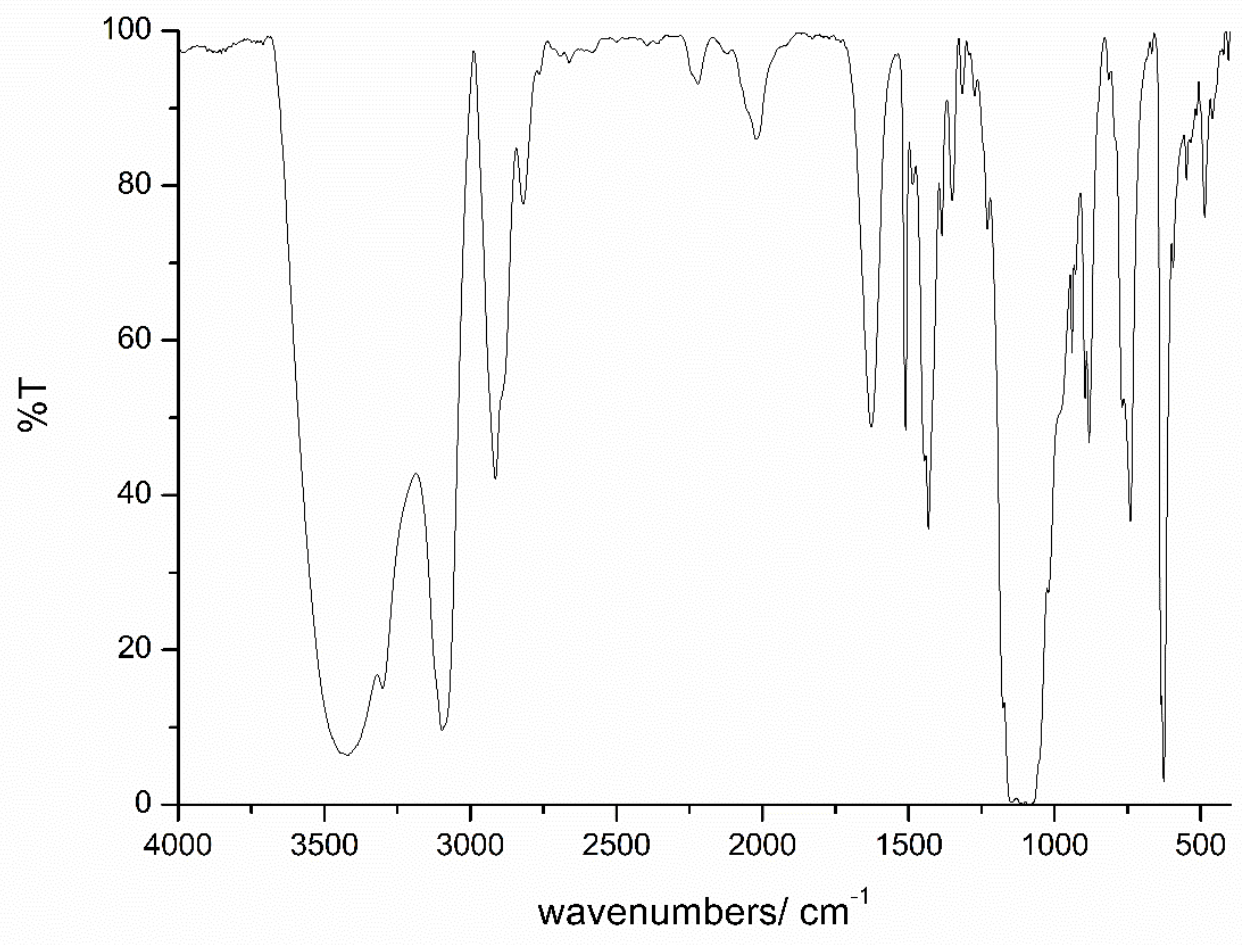

Figure S13: IR spectrum of dried $\left[\mathrm{Fe}^{\mathrm{II}}{ }_{2}\left(\mu_{2}-\mathrm{L}^{2}\right)_{2}\right]\left(\mathrm{ClO}_{4}\right)_{4}(\mathbf{C 5})$.

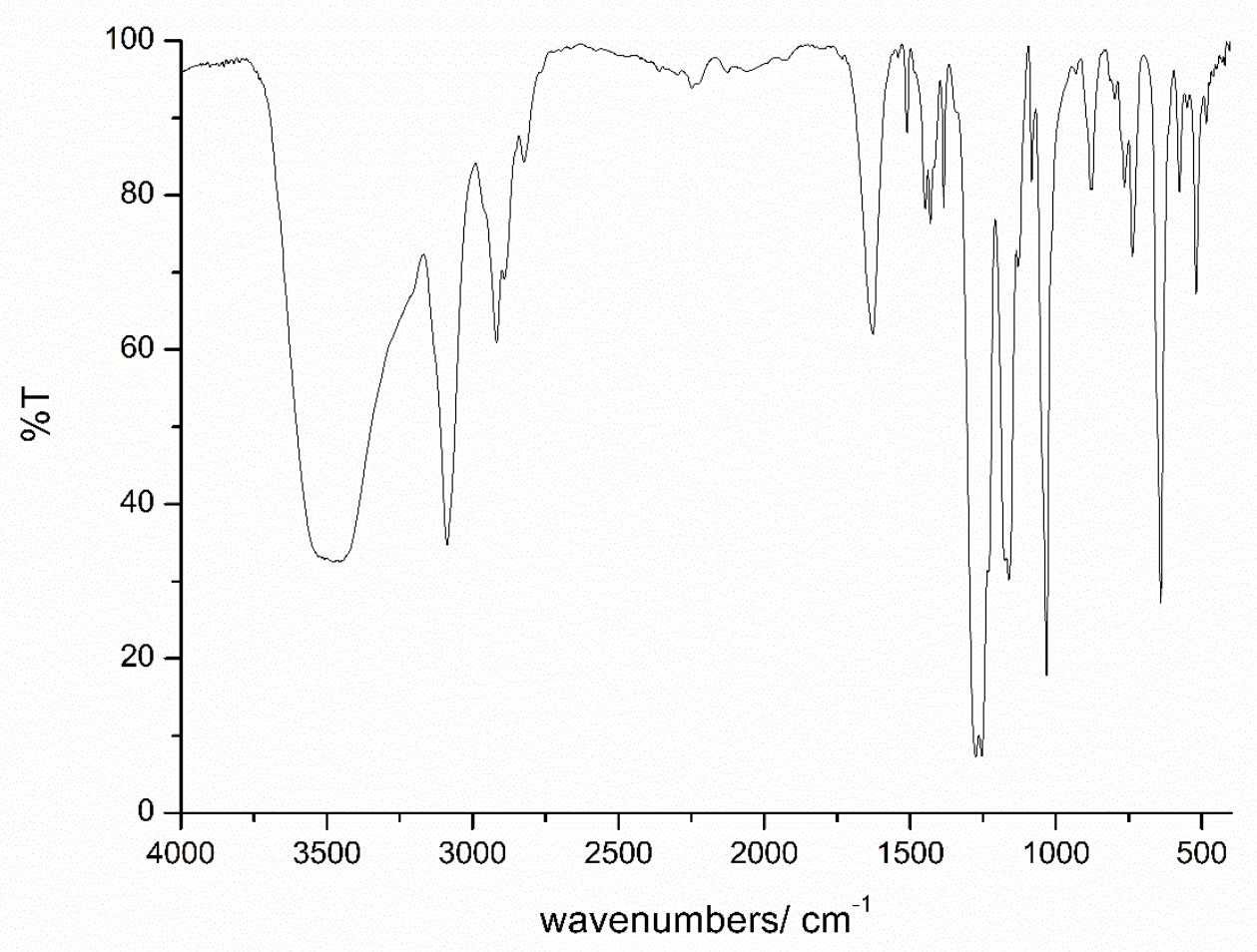

Figure S14: IR spectrum of dried $\left[\mathrm{Fe}^{\mathrm{II}}{ }_{2}\left(\mu_{2}-\mathrm{L}^{2}\right)_{2}\right]\left(\mathrm{F}_{3} \mathrm{CSO}_{3}\right)_{4}$ (C6). 
X-ray diffraction measurements

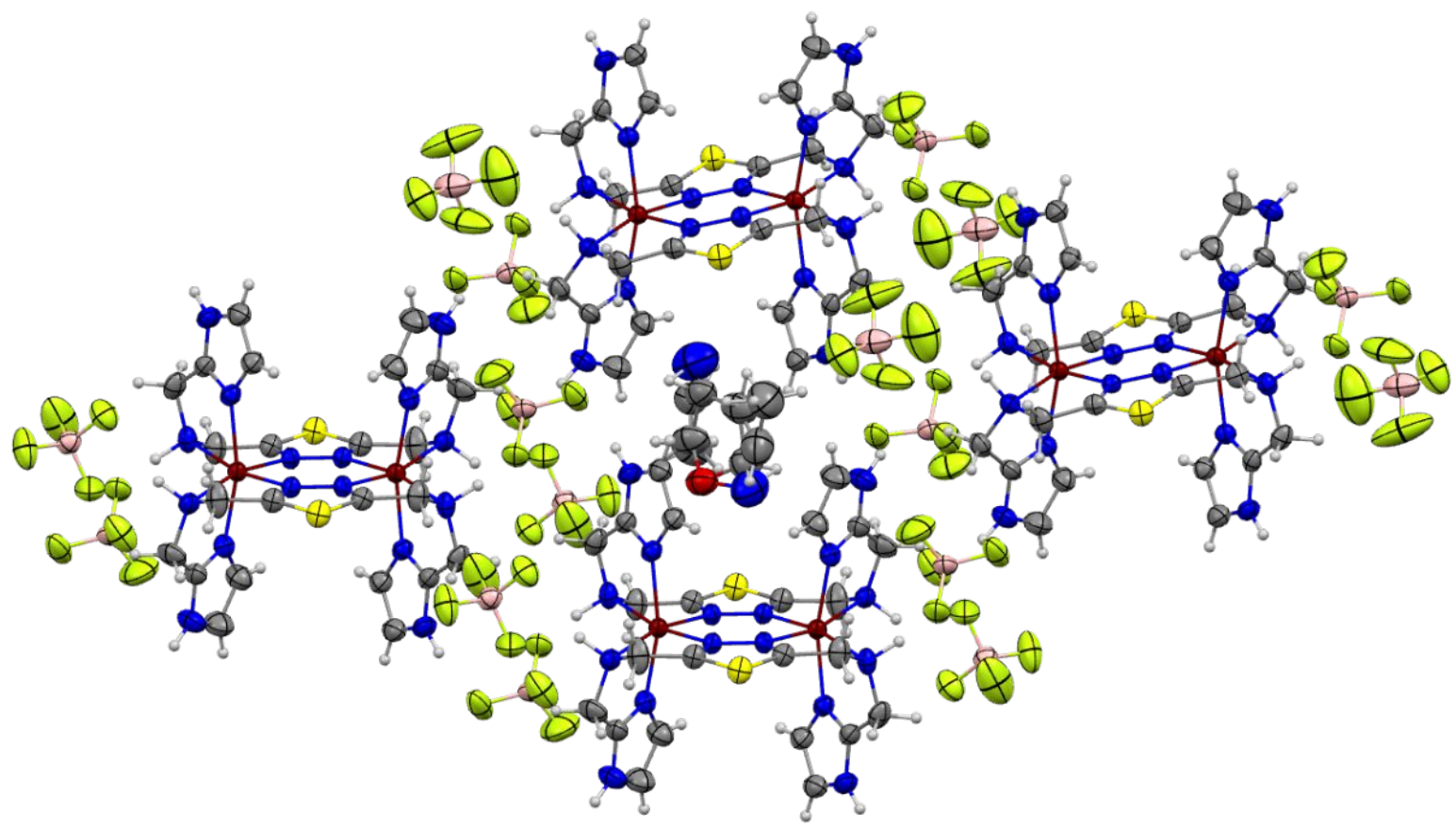

Figure S15: Packing of the molecules in complex $\left[\mathrm{Fe}^{\mathrm{I}}{ }_{2}\left(\mu_{2}-\mathrm{L}^{1}\right)_{2}\right]\left(\mathrm{BF}_{4}\right)_{4} * 0.67 \mathrm{THF} * 0.65 \mathrm{MeCN}(\mathbf{C} 1)$ with thermal ellipsoids. Color code: Fe dark red, $\mathrm{N}$ blue, $\mathrm{S}$ yellow, $\mathrm{C}$ grey, $\mathrm{H}$ white, $\mathrm{O}$ red, B pink and $\mathrm{F}$ green.
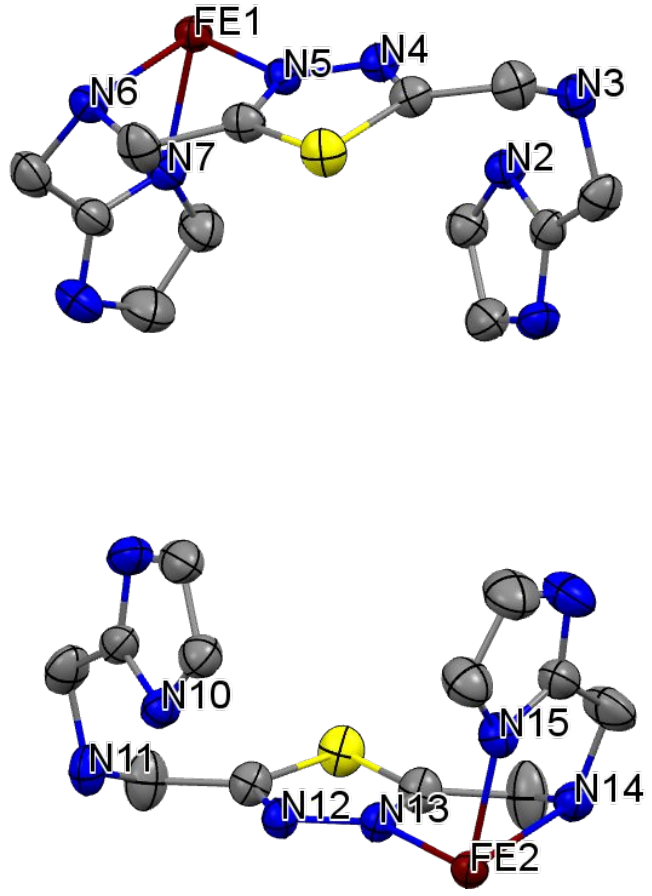

Figure S16: Asymmetric unit of $\left[\mathrm{Fe}_{2}{ }_{2}\left(\mu_{2}-\mathrm{L}^{1}\right)_{2}\right]\left(\mathrm{BF}_{4}\right)_{4} * 0.67 \mathrm{THF} * 0.65 \mathrm{MeCN}(\mathrm{C1})$ without hydrogens, solvent molecules and counter ions. Color code: Fe dark red, $\mathrm{N}$ blue, $\mathrm{S}$ yellow and $\mathrm{C}$ grey. 


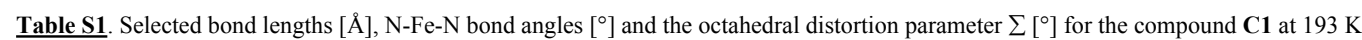

\begin{tabular}{|c|c|}
\hline & C1 (@193 K) \\
\hline $\mathrm{Fe}-\mathrm{N}_{\mathrm{TDA}}{ }^{[\mathrm{a}]}$ & $\begin{array}{l}2.150(3), 2.185(3) / \\
2.169(3), 2.172(3)\end{array}$ \\
\hline $\mathrm{Fe}-\mathrm{N}_{\mathrm{Imz} / \mathrm{Ta}}{ }^{[\mathrm{a}]}$ & $\begin{array}{c}2.096(4), 2.111(4) / \\
2.084(4), 2.090(4)\end{array}$ \\
\hline $\mathrm{Fe}-\mathrm{N}_{\mathrm{NH}}{ }^{[\mathrm{a}]}$ & $\begin{array}{l}2.313(3), 2.342(3) / \\
2.321(3), 2.343(4)\end{array}$ \\
\hline av. Fe-N & $2.200 / 2.196$ \\
\hline av. cis $\mathrm{N}-\mathrm{Fe}-\mathrm{N}^{[\mathrm{a}]}$ & $90.3 / 90.4$ \\
\hline av. trans $\mathrm{N}-\mathrm{Fe}-\mathrm{N}^{[\mathrm{a}]}$ & $167.1 / 167.0$ \\
\hline$\sum^{[\mathrm{a}][\mathrm{b}]}$ & $115.26 / 115.30$ \\
\hline
\end{tabular}

[a] Fe1/Fe2. [b] Octahedral distortion parameter $\Sigma$ (sum of the deviation from $90^{\circ}$ of the 12 cis-N-Fe-N angles in the FeN6 coordination sphere) 


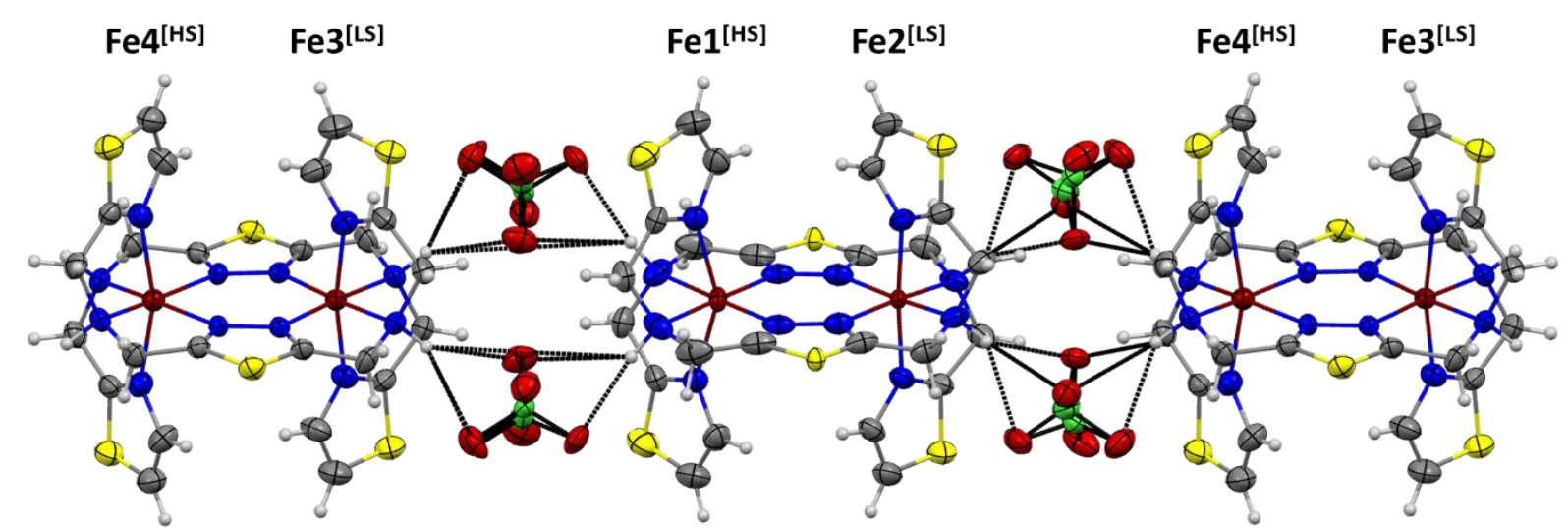

Figure S17: Representation of the 1D chain for C5 (@160 K, slow cooling). The dimers along the chain are ordered in the same way ([HS-LS]•[HS-LS]•[HS-LS]). This figure is representative for all formed 1D chains with the different counter ions $(\mathbf{C} 4-\mathbf{C 6})$ although the intermolecular distances differ. Solvent molecules and non-bridging counter ions have been omitted for clarity. Hydrogen bonds are shown as black dashed lines. Color code: Fe dark red, $\mathrm{N}$ blue, $\mathrm{S}$ yellow, $\mathrm{C}$ grey, $\mathrm{H}$ white, $\mathrm{O}$ red and $\mathrm{Cl}$ green.
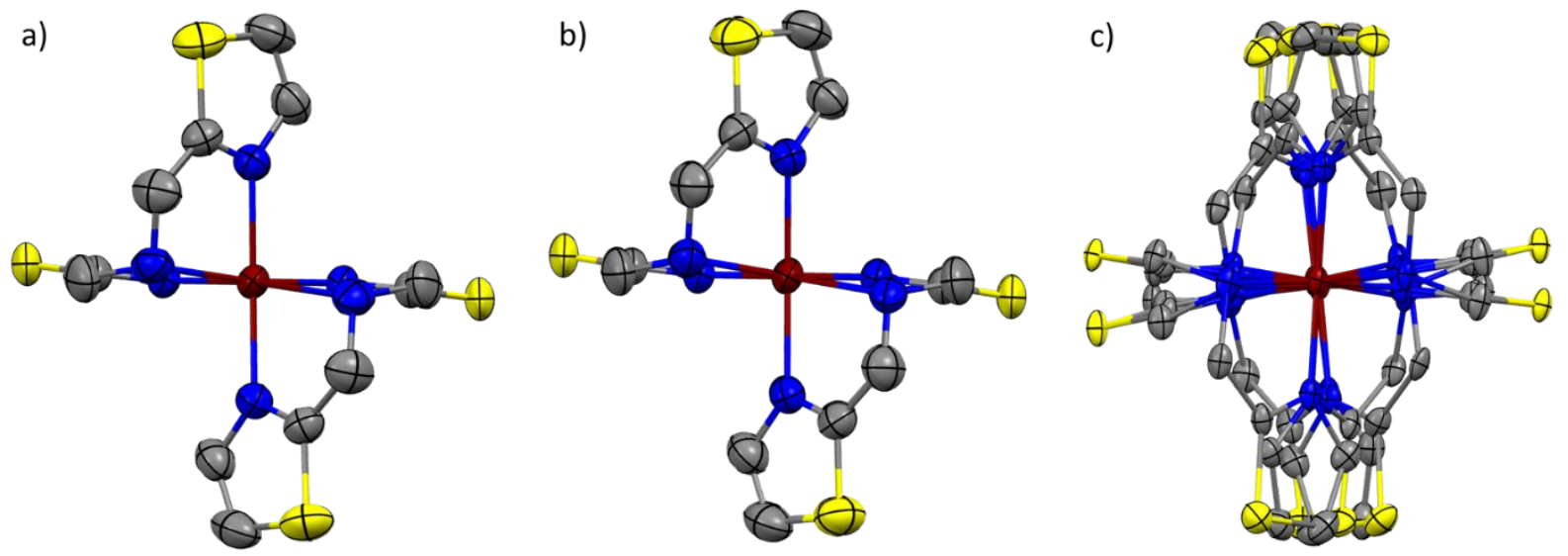

Figure S18: Different orientation of the complex cations of a) $\left[\mathrm{Fe}_{2}{ }_{2}\left(\mu_{2}-\mathrm{L}^{2}\right)_{2}\right]\left(\mathrm{BF}_{4}\right)_{4} * 3 \mathrm{MeCN}(\mathrm{C} 4)$, b) $\left[\mathrm{Fe}^{\mathrm{II}}{ }_{2}\left(\mu_{2}-\mathrm{L}^{2}\right)_{2}\right]\left(\mathrm{ClO}_{4}\right)_{4} * 3 \mathrm{MeCN}(\mathbf{C 5})$ and c) $\left[\mathrm{Fe}_{2}{ }_{2}\left(\mu_{2}-\mathrm{L}^{2}\right)_{2}\right]\left(\mathrm{F}_{3} \mathrm{CSO}_{3}\right)_{4} * 2 \mathrm{MeCN}(\mathbf{C 6})$ along the 1D chain. Hydrogens, solvent molecules and counter ions have been omitted for clarity. Color code: Fe dark red, $\mathrm{N}$ blue, $\mathrm{S}$ yellow and $\mathrm{C}$ grey. 
a)

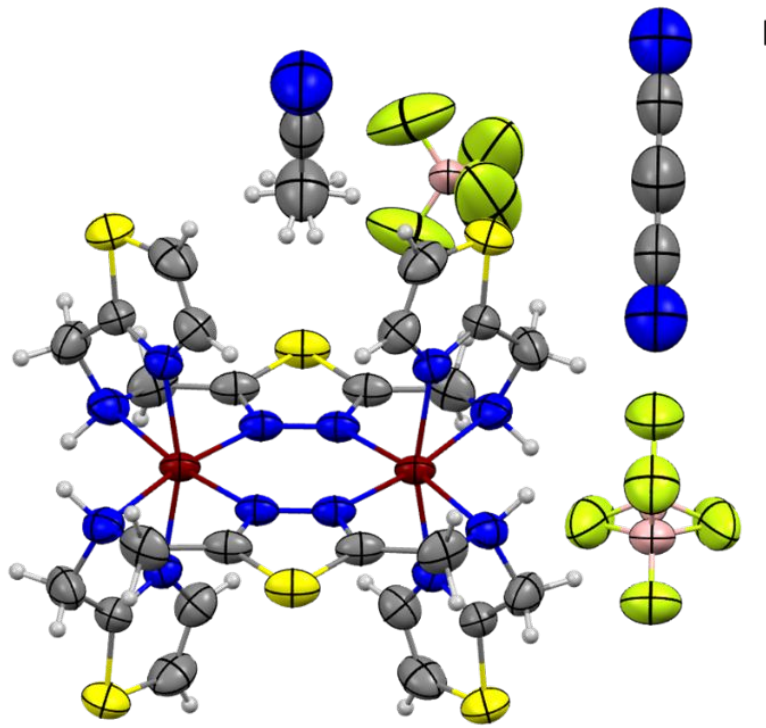

b)

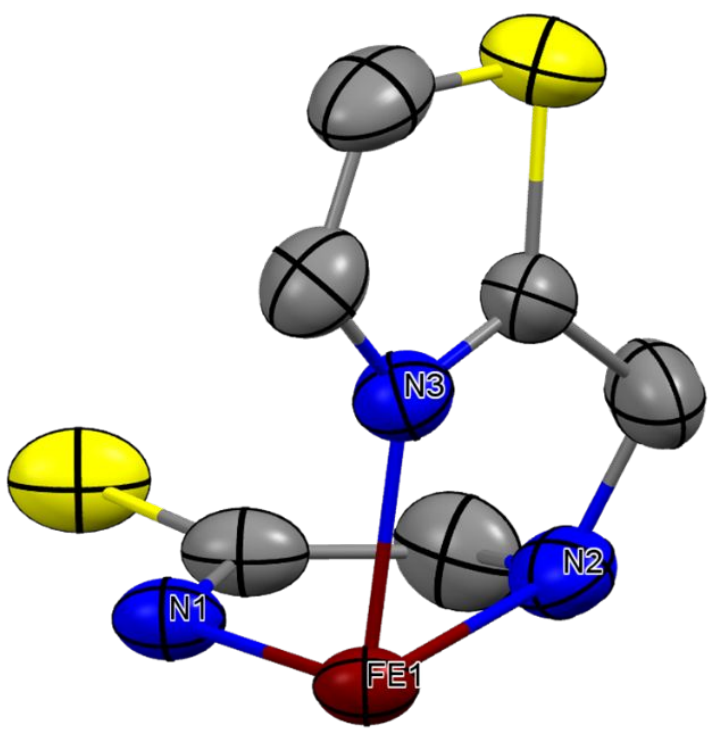

Figure S19: a) Molecular structure of $\left[\mathrm{Fe}_{2}{ }_{2}\left(\mu_{2}-\mathrm{L}^{2}\right)_{2}\right]\left(\mathrm{BF}_{4}\right)_{4} * 3 \mathrm{MeCN}(\mathbf{C 4})$ with thermal ellipsoids at $240 \mathrm{~K}$. b) Asymmetric unit without hydrogens, solvent molecules and counter ions. Color code: $\mathrm{Fe}$ is dark red, $\mathrm{N}$ blue, $\mathrm{S}$ yellow, $\mathrm{C}$ grey, $\mathrm{H}$ white, $\mathrm{B}$ pink and $\mathrm{F}$ green.

a)

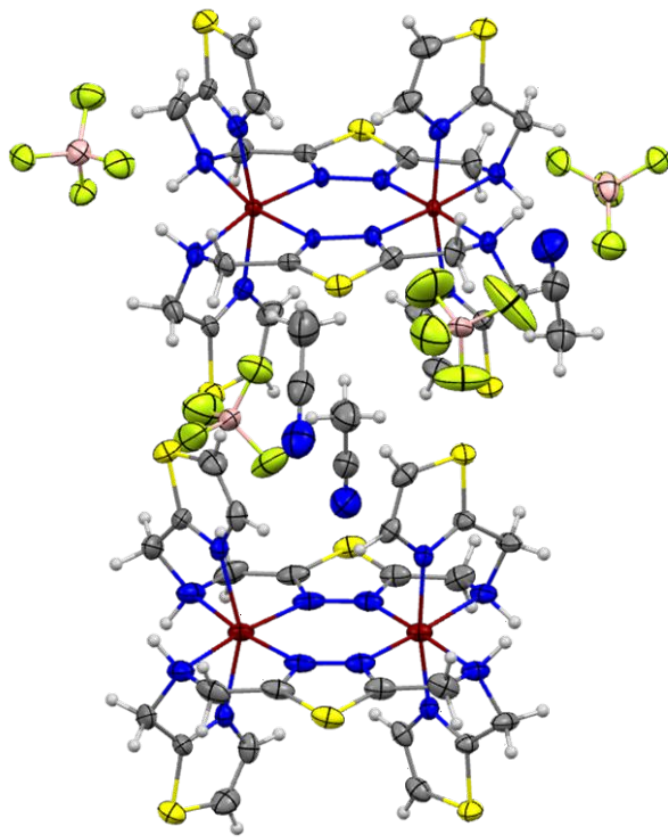

b)

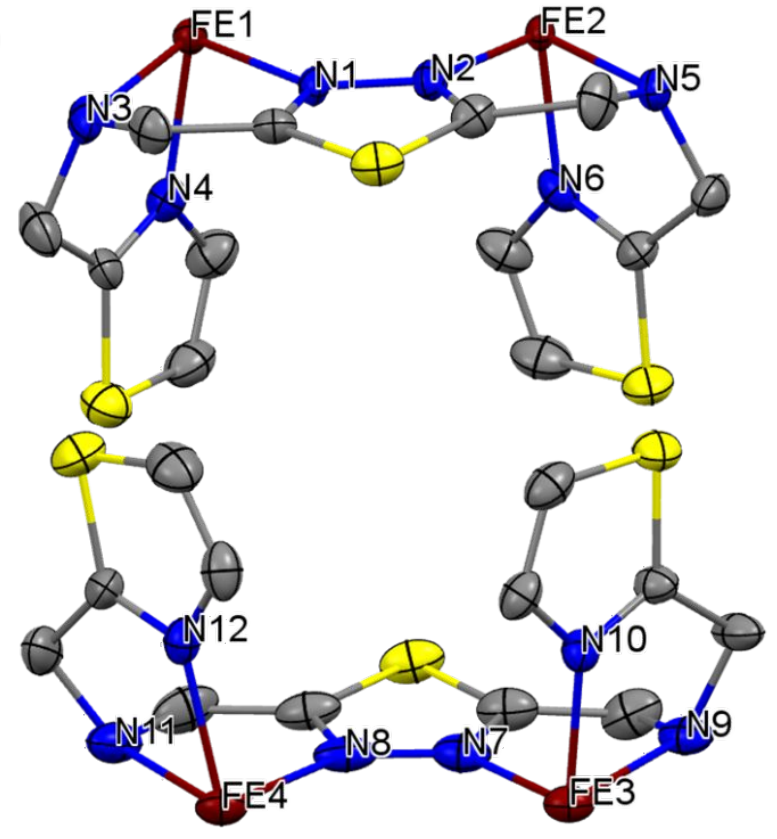

Figure S20: a) Molecular structure of $\left[\mathrm{Fe}^{\mathrm{II}}\left(\mu_{2}-\mathrm{L}^{2}\right)_{2}\right]\left(\mathrm{BF}_{4}\right)_{4} * 3 \mathrm{MeCN}(\mathbf{C 4})$ with thermal ellipsoids at $120 \mathrm{~K}$. b) Asymmetric unit without hydrogens, solvent molecules and counter ions. Color code: Fe dark red, $\mathrm{N}$ blue, $\mathrm{S}$ yellow, $\mathrm{C}$ grey, $\mathrm{H}$ white, B pink and $\mathrm{F}$ green. 
a)

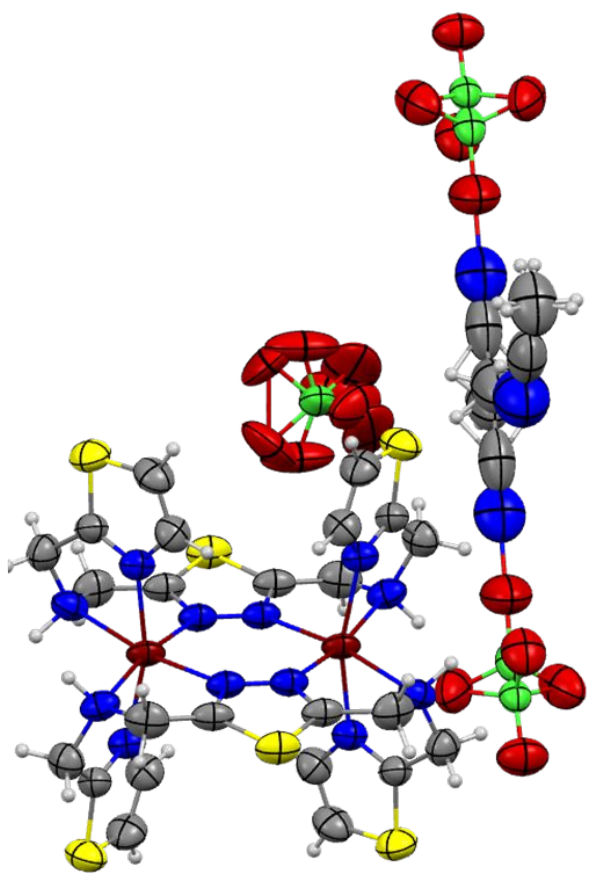

b)

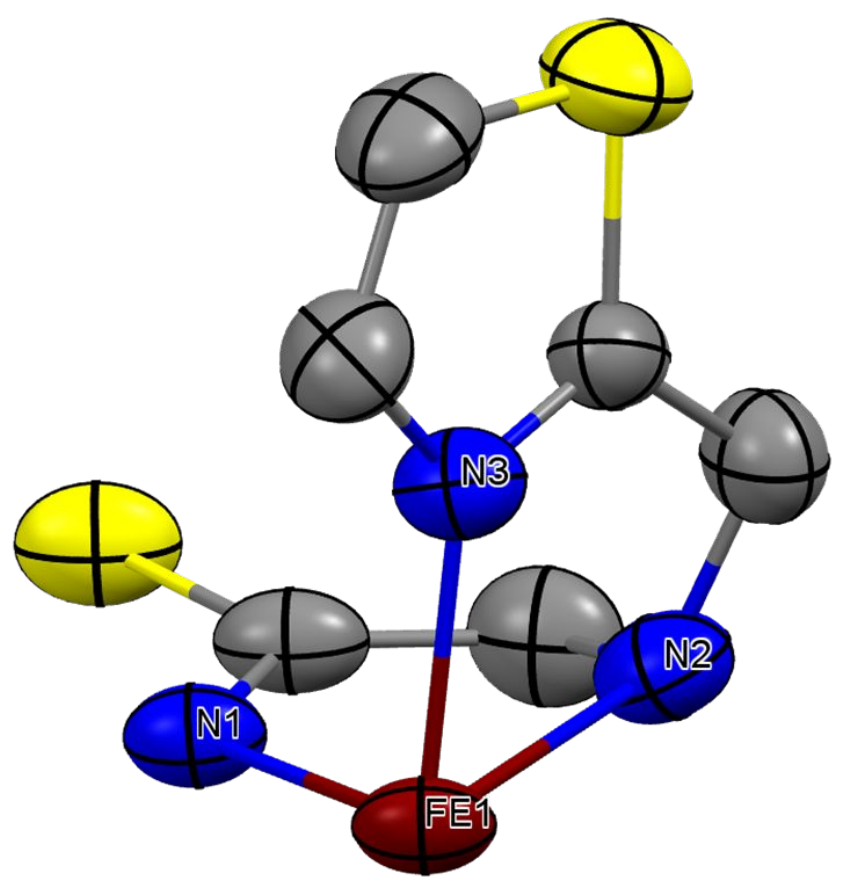

Figure S21: a) Molecular structure of $\left[\mathrm{Fe}_{2}{ }_{2}\left(\mu_{2}-\mathrm{L}^{2}\right)_{2}\right]\left(\mathrm{ClO}_{4}\right)_{4} * 3 \mathrm{MeCN}(\mathbf{C 5})$ with thermal ellipsoids at $250 \mathrm{~K}$. b) Asymmetric unit without hydrogens, solvent molecules and counter ions. Color code: $\mathrm{Fe}$ dark red, $\mathrm{N}$ blue, $\mathrm{S}$ yellow, $\mathrm{C}$ grey, $\mathrm{H}$ white, $\mathrm{O}$ red and $\mathrm{Cl}$ green.

a)

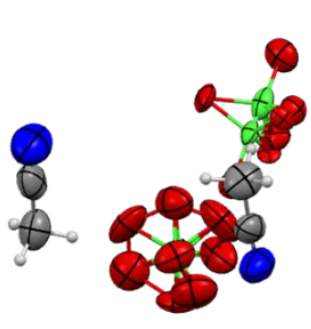

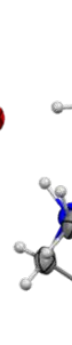

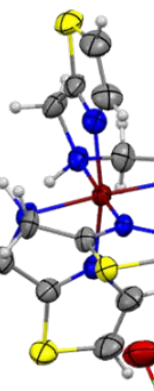

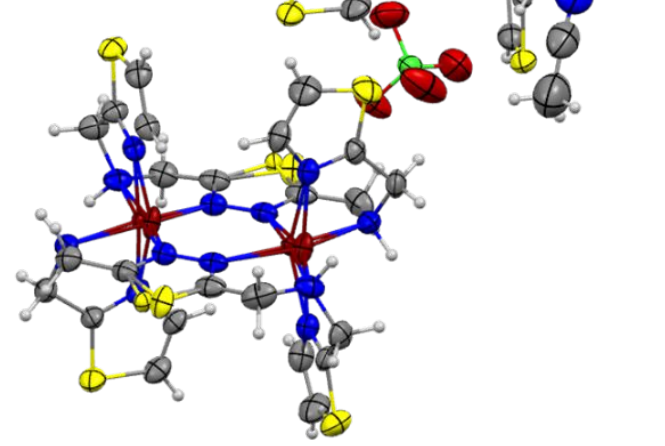

b)
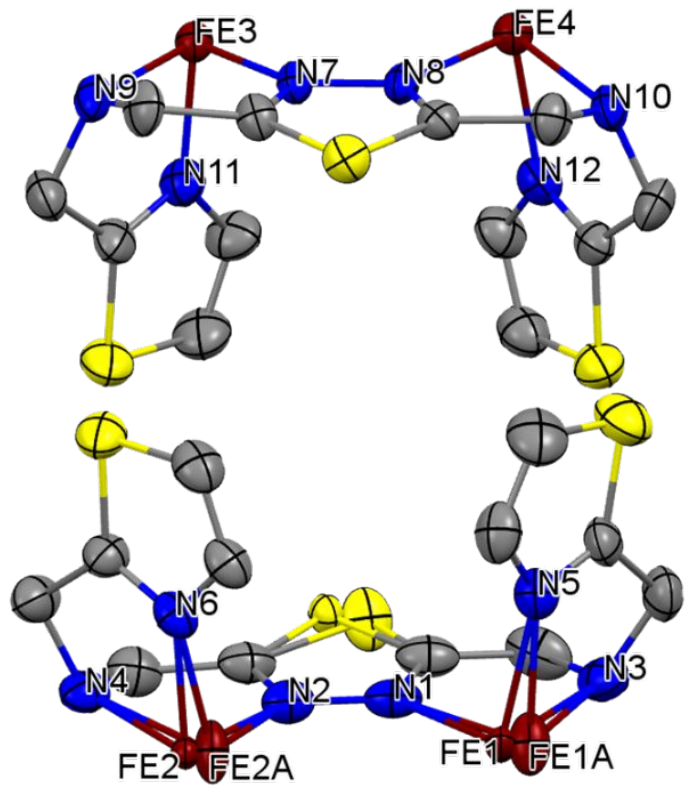

Figure S22: a) Molecular structure of $\left[\mathrm{Fe}_{2}{ }_{2}\left(\mu_{2}-\mathrm{L}^{2}\right)_{2}\right]\left(\mathrm{ClO}_{4}\right)_{4} * 3 \mathrm{MeCN}(\mathrm{C5})$ with thermal ellipsoids at $160 \mathrm{~K}$ after slow cooling. b) Asymmetric unit without hydrogens, solvent molecules and counter ions. Color code: $\mathrm{Fe}$ dark red, $\mathrm{N}$ blue, $\mathrm{S}$ yellow, $\mathrm{C}$ grey, $\mathrm{H}$ white, $\mathrm{O}$ red and $\mathrm{Cl}$ green. 


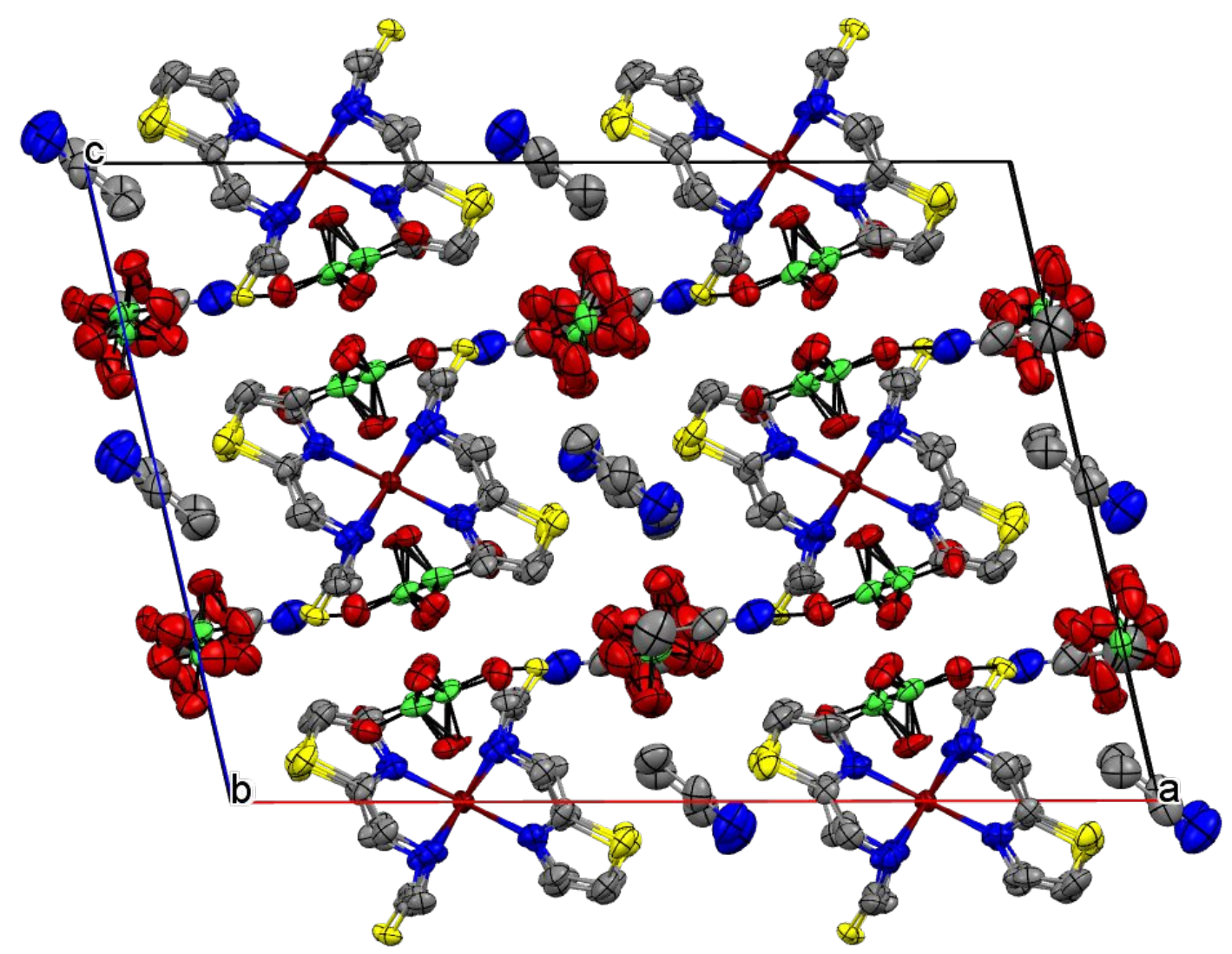

Figure S23: Packing of $\left[\mathrm{Fe}_{2}{ }_{2}\left(\mu_{2}-\mathrm{L}^{2}\right)_{2}\right]\left(\mathrm{ClO}_{4}\right)_{4} * 3 \mathrm{MeCN}(\mathbf{C 5})$ along b-axis with thermal ellipsoids at $160 \mathrm{~K}$ after slow cooling. Hydrogens have been omitted for clarity. Color code: $\mathrm{Fe}$ dark red, $\mathrm{N}$ blue, $\mathrm{S}$ yellow, $\mathrm{C}$ grey, $\mathrm{O}$ red and $\mathrm{Cl}$ green.

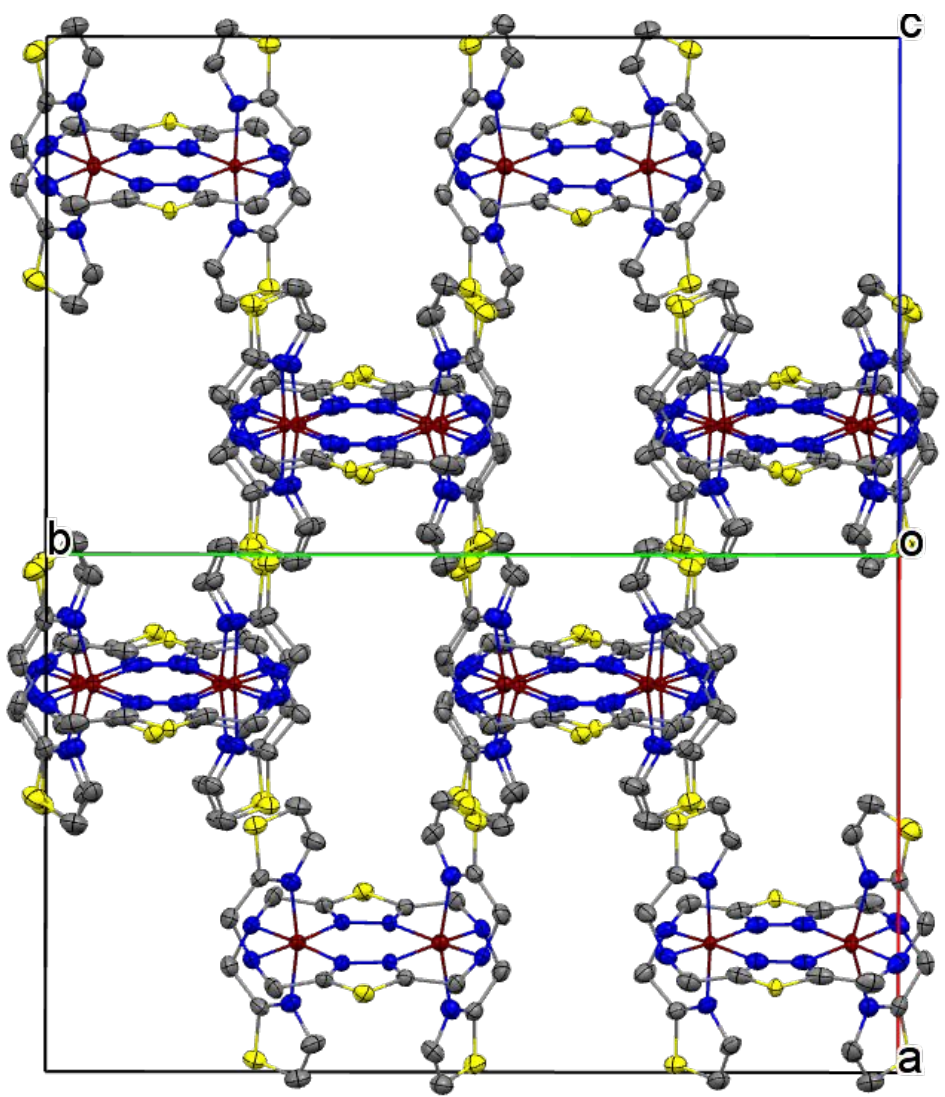

Figure S24: Packing of $\left[\mathrm{Fe}^{\mathrm{II}}\left(\mu_{2}-\mathrm{L}^{2}\right)_{2}\right]\left(\mathrm{ClO}_{4}\right)_{4} * 3 \mathrm{MeCN}$ (C5) along the bisecting between a and $\mathrm{c}$ with thermal ellipsoids at $160 \mathrm{~K}$ after slow cooling. Hydrogens, solvent molecules and counter ions have been omitted for clarity. Color code: Fe dark red, $\mathrm{N}$ blue, $\mathrm{S}$ yellow, $\mathrm{C}$ grey. 
a)

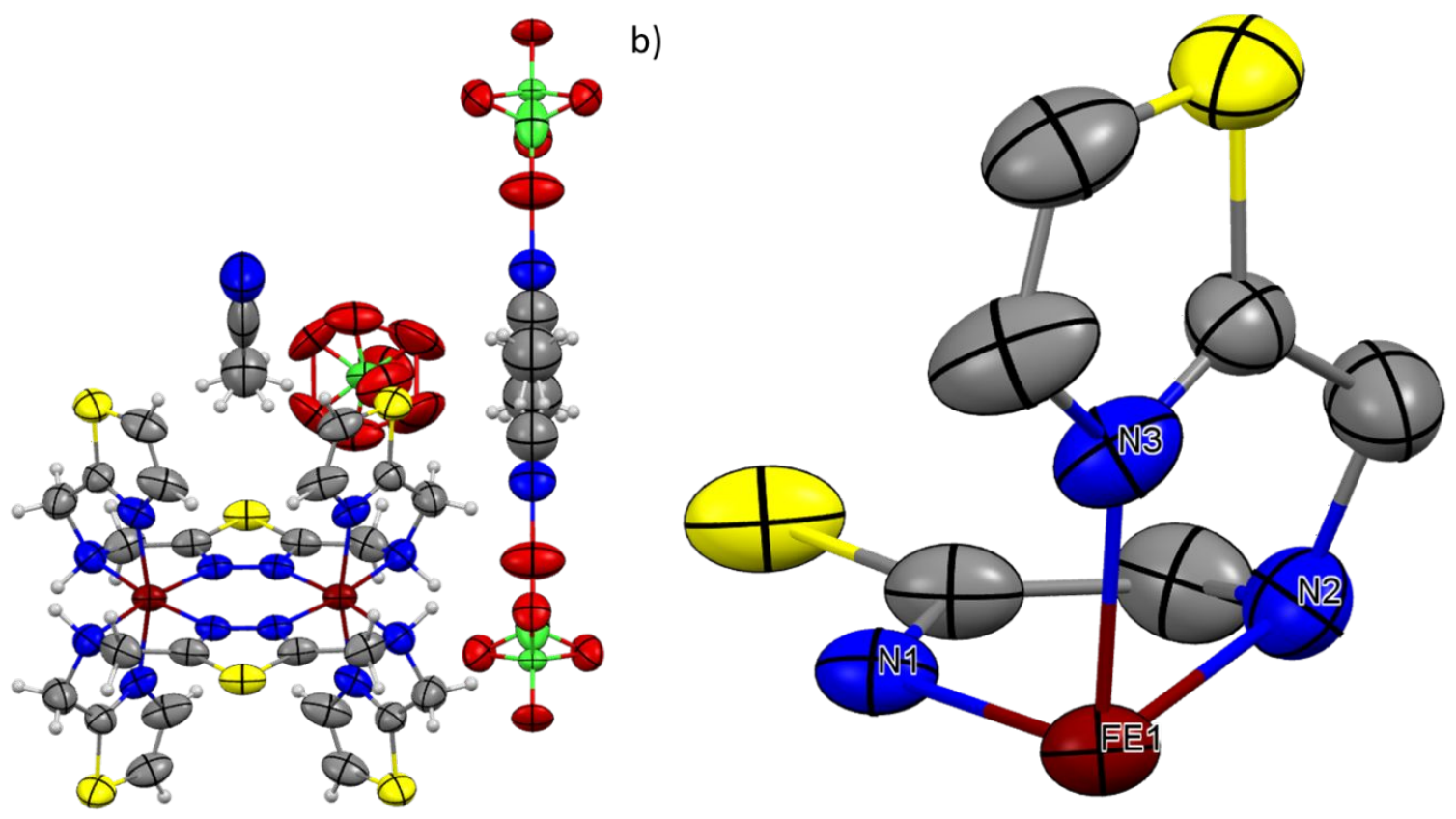

Figure S25: a) Molecular structure of $\left[\mathrm{Fe}^{\mathrm{II}}{ }_{2}\left(\mu_{2}-\mathrm{L}^{2}\right)_{2}\right]\left(\mathrm{ClO}_{4}\right)_{4} * 3 \mathrm{MeCN}(\mathbf{C 5})$ with thermal ellipsoids at $160 \mathrm{~K}$ after quenching. b) Asymmetric unit without hydrogens, solvent molecules and counter ions. Color code: Fe dark red, $\mathrm{N}$ blue, $\mathrm{S}$ yellow, $\mathrm{C}$ grey, $\mathrm{H}$ white, $\mathrm{O}$ red and $\mathrm{Cl}$ green.

a)

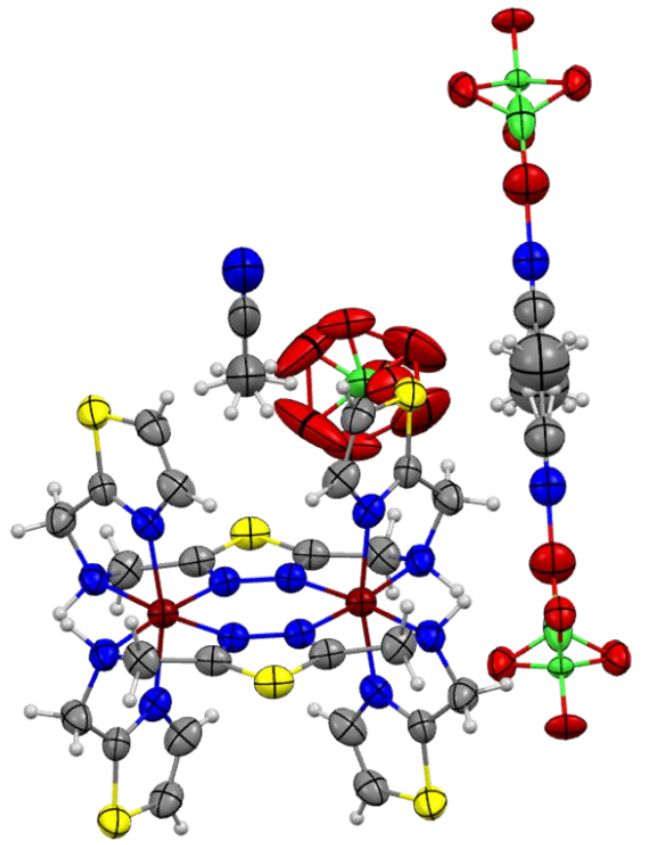

b)

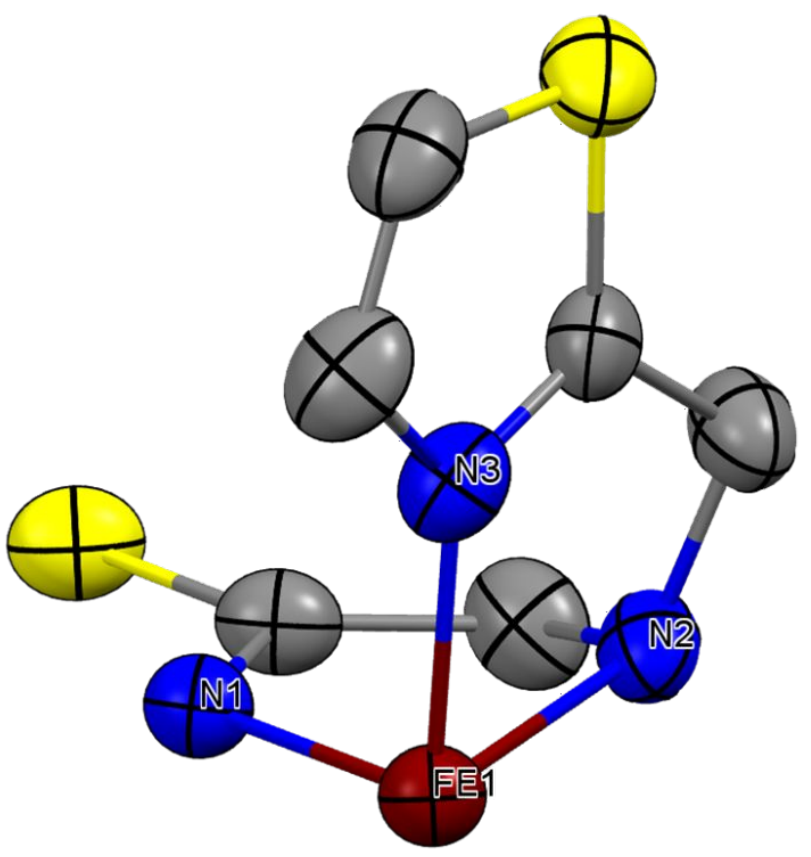

Figure S26: a)Molecular structure of $\left[\mathrm{Fe}^{\mathrm{II}}{ }_{2}\left(\mu_{2}-\mathrm{L}^{2}\right)_{2}\right]\left(\mathrm{ClO}_{4}\right)_{4} * 3 \mathrm{MeCN}(\mathbf{C 5})$ with thermal ellipsoids at $100 \mathrm{~K}$. b) Asymmetric unit without hydrogens, solvent molecules and counter ions. Color code: $\mathrm{Fe}$ dark red, $\mathrm{N}$ blue, $\mathrm{S}$ yellow, $\mathrm{C}$ grey, $\mathrm{H}$ white, $\mathrm{O}$ red and $\mathrm{Cl}$ green. 

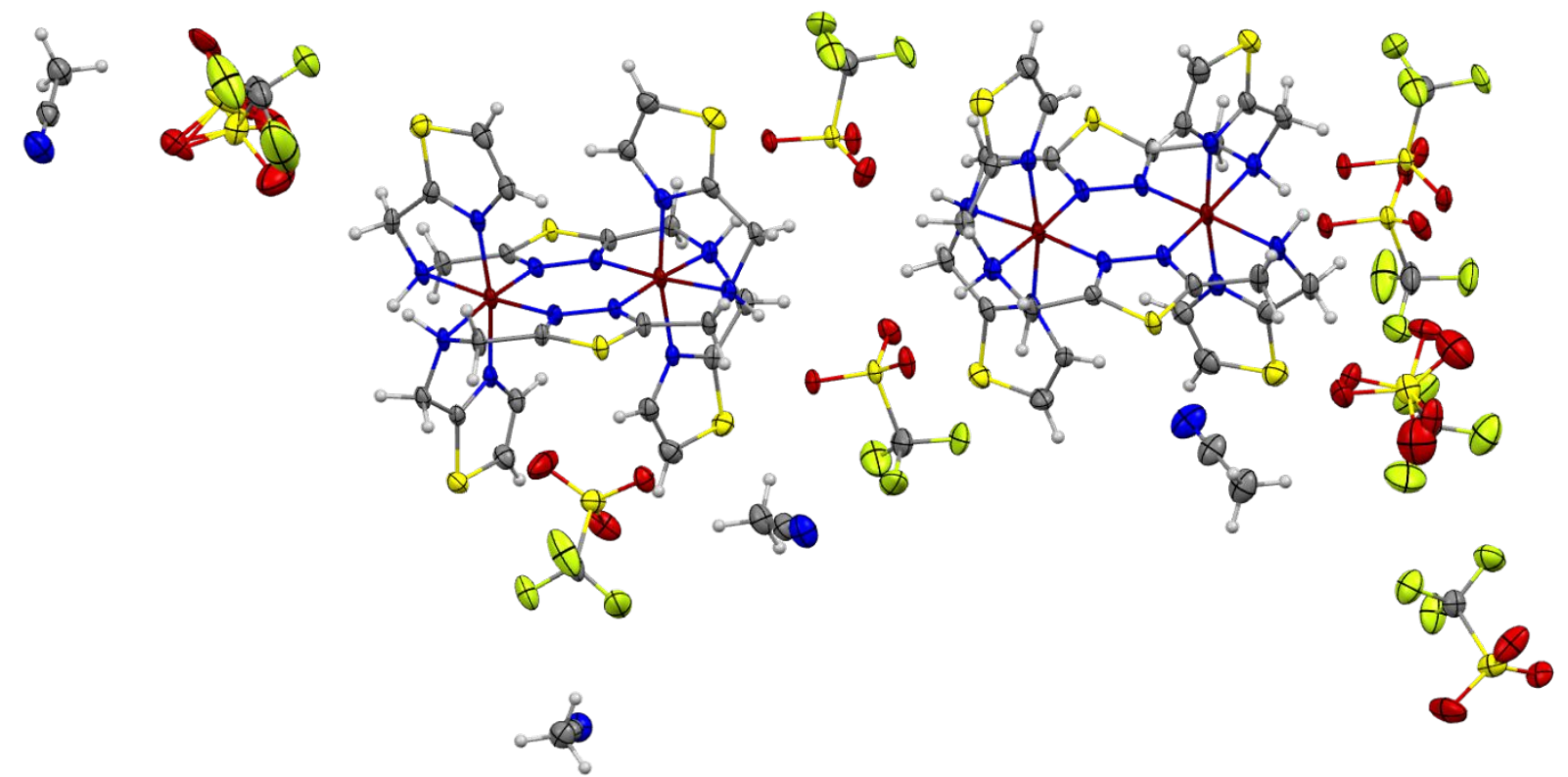

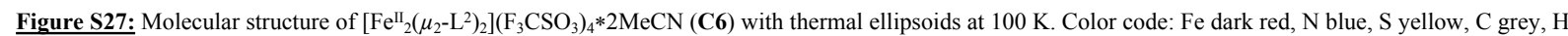
white, $\mathrm{O}$ red and $\mathrm{Cl}$ green.
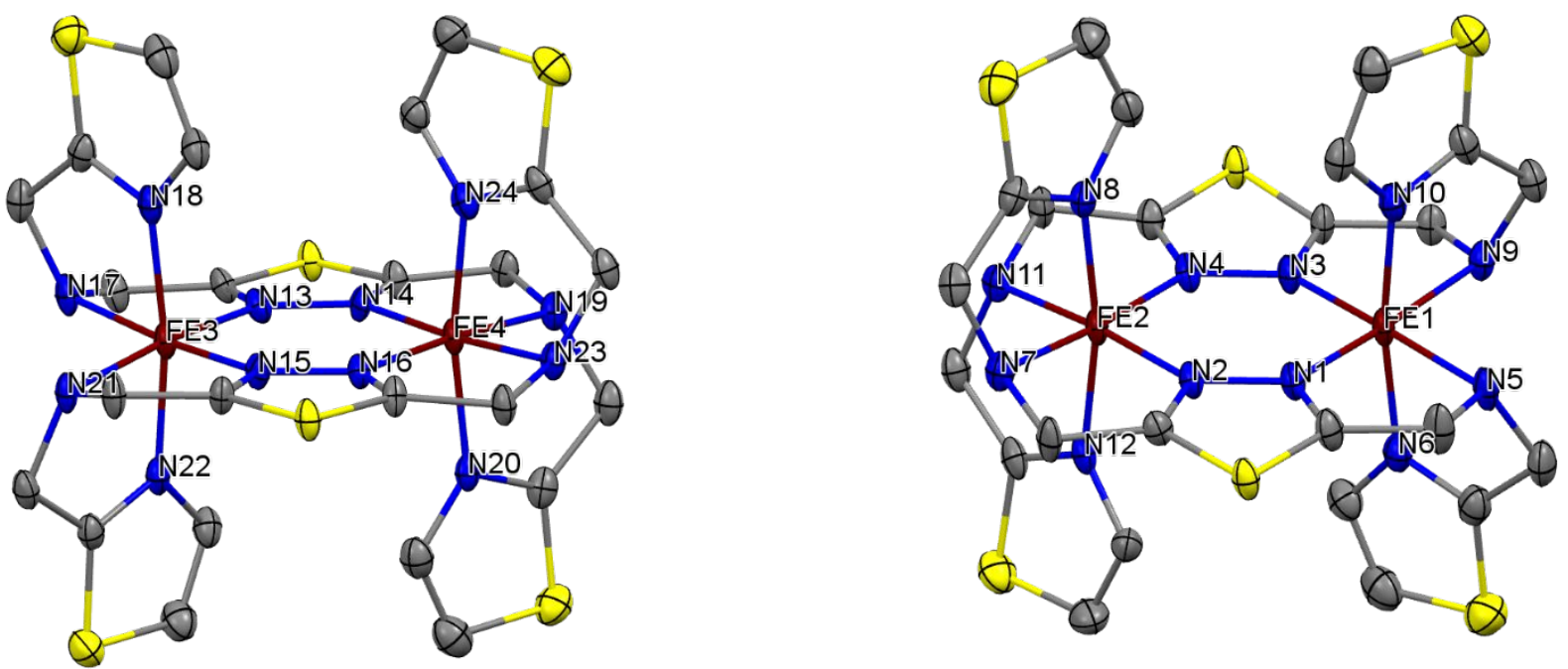

Figure S28: Asymmetric unit of $\left[\mathrm{Fe}_{2}{ }_{2}\left(\mu_{2}-\mathrm{L}^{2}\right)_{2}\right]\left(\mathrm{F}_{3} \mathrm{CSO}_{3}\right)_{4} * 2 \mathrm{MeCN}(\mathbf{C 6})$ without Hydrogens, solvent molecules and counter ions. Color code: $\mathrm{Fe}$ dark red, $\mathrm{N}$ blue, $\mathrm{S}$ yellow and $\mathrm{C}$ grey. 
Table S2: Crystallographic parameters for all discussed crystal structures of C1 - C6.

\begin{tabular}{|c|c|c|c|c|c|c|c|c|c|}
\hline & C1 & C4 (@120k) & C4 (@240k) & C5 (@100K) & C5 (@160K) - 1 & C5 (@160K) - & C5 (@160K) - 2 & C5 (@250K) & C6 \\
\hline formula & $\begin{array}{c}\mathrm{C} 28 \mathrm{H} 39.34 \mathrm{~B} 4 \mathrm{~F} 16 \\
\mathrm{Fe} 2 \mathrm{~N} 16.65 \text { O0.67 S2 }\end{array}$ & $\begin{array}{c}\text { C60 H74 B8 } \\
\text { F32 Fe4 N30 } \\
\text { S12 }\end{array}$ & $\begin{array}{c}\text { C30 H37 B4 } \\
\text { F16 Fe3 N15 S6 }\end{array}$ & $\begin{array}{c}\mathrm{C} 30 \mathrm{H} 37 \mathrm{Cl} 4 \\
\mathrm{Fe} 2 \mathrm{~N} 15 \mathrm{O} 16 \\
\mathrm{~S} 6\end{array}$ & $\begin{array}{c}\mathrm{C} 60 \mathrm{H} 74 \mathrm{Cl} 8 \\
\mathrm{Fe} 4 \mathrm{~N} 30 \mathrm{O} 32 \\
\text { S12 }\end{array}$ & $\begin{array}{c}\mathrm{C} 60 \mathrm{H} 74 \mathrm{Cl} 8 \\
\mathrm{Fe} 4 \mathrm{~N} 30 \mathrm{O} 32 \\
\mathrm{~S} 12\end{array}$ & $\begin{array}{c}\mathrm{C} 30 \mathrm{H} 37 \mathrm{Cl} 4 \\
\mathrm{Fe} 2 \mathrm{~N} 15 \mathrm{O} 16 \\
\text { S6 }\end{array}$ & $\begin{array}{c}\mathrm{C} 30 \mathrm{H} 37 \mathrm{Cl} 4 \\
\mathrm{Fe} 2 \mathrm{~N} 15 \mathrm{O} 16 \\
\text { S6 }\end{array}$ & $\begin{array}{c}\text { C64 H68 F24 Fe4 } \\
\text { N28 O24 S20 }\end{array}$ \\
\hline molar weigth $[\mathrm{g} / \mathrm{mol}]$ & 1143.13 & 2518.09 & 1259.04 & 1309.6 & 2619.21 & 2619.21 & 1309.6 & 1309.6 & 2934.06 \\
\hline crystal system & triclinic & monoclinic & monoclinic & monoclinic & monoclinic & monoclinic & monoclinic & monoclinic & Triclinic \\
\hline space group & $P-1$ & $I 2 / a$ & $I 2 / m$ & $C 2 / m$ & $I 2 / a$ & $C 2 / c$ & $C 2 / m$ & $C 2 / m$ & $P-1$ \\
\hline $\mathrm{a} / \AA$ & $12.0375(7)$ & $16.7167(7)$ & $13.004(3)$ & $17.0993(10)$ & $23.9111(8)$ & $26.0447(9)$ & $17.006(2)$ & $16.9633(18)$ & $14.1442(16)$ \\
\hline $\mathrm{b} / \AA \AA$ & $13.0822(7)$ & $24.8024(13)$ & $12.609(3)$ & $12.3564(9)$ & $24.9799(10)$ & $24.9799(10)$ & $12.4677(17)$ & $12.6415(17)$ & $27.6210(19)$ \\
\hline $\mathrm{c} / \AA$ & $14.6807(8)$ & $23.5826(11)$ & $16.117(3)$ & $12.8089(8)$ & $16.9439(6)$ & $16.9439(6)$ & $12.898(2)$ & $13.2025(14)$ & $23.975(3)$ \\
\hline$\alpha /^{\circ}$ & 91.122(4) & 90 & 90 & 90 & 90 & 90 & 90 & 90 & 96.402(9) \\
\hline$\beta /^{\circ}$ & $103.133(4)$ & $103.305(4)$ & 110.83(3) & $116.817(4)$ & $102.872(3)$ & $116.490(3)$ & $116.051(10)$ & $115.795(7)$ & $101.108(8)$ \\
\hline$\gamma /{ }^{\circ}$ & $98.453(3)$ & 90 & 90 & 90 & 90 & 90 & 90 & 90 & $110.374(10)$ \\
\hline $\mathrm{V} / \AA^{3}$ & $2223.6(2)$ & $9515.2(8)$ & $2469.8(10)$ & 2415.3(3) & $9866.2(6)$ & $9866.3(7)$ & 2456.9 & $2549.1(5)$ & $5392.1(11)$ \\
\hline $\mathrm{Z}$ & 2 & 4 & 2 & 2 & 4 & 4 & 2 & 2 & 4 \\
\hline $\mathrm{T} / \mathrm{K}$ & 193(2) & $120(2)$ & $240(2)$ & $100(2)$ & $160(2)$ & $160(2)$ & $160(2)$ & $250(2)$ & $100(2)$ \\
\hline$\rho_{\text {calcd. }}\left[\mathrm{g} / \mathrm{cm}^{3}\right]$ & 1.707 & 1.758 & 1.693 & 1.801 & 1.763 & 1.763 & 1.77 & 1.706 & 1.807 \\
\hline$\mu\left[\mathrm{mm}^{-1}\right]$ & 0.863 & 0.982 & 0.946 & 1.165 & 1.141 & 1.141 & 1.145 & 1.104 & 1.036 \\
\hline$R$ (int) & 0.0324 & 0.025 & 0.0184 & 0.0265 & 0.0361 & 0.0361 & 0.0394 & 0.0173 & 0.0543 \\
\hline $\mathrm{S}$ & 1.027 & 1.07 & 1.046 & 1.123 & 1.05 & 1.040 & 1.141 & 1.09 & 1.03 \\
\hline $\mathrm{R} 1(1>2 \sigma(1))$ & 0.612 & 0.1067 & 0.0616 & 0.074 & 0.0921 & 0.0922 & 0.0978 & 0.0525 & 0.0626 \\
\hline$w R 2$ (all data) & 0.1843 & 0.3077 & 0.1794 & 0.1859 & 0.2777 & 0.2817 & 0.2471 & 0.1586 & 0.1714 \\
\hline
\end{tabular}


Magnetic data

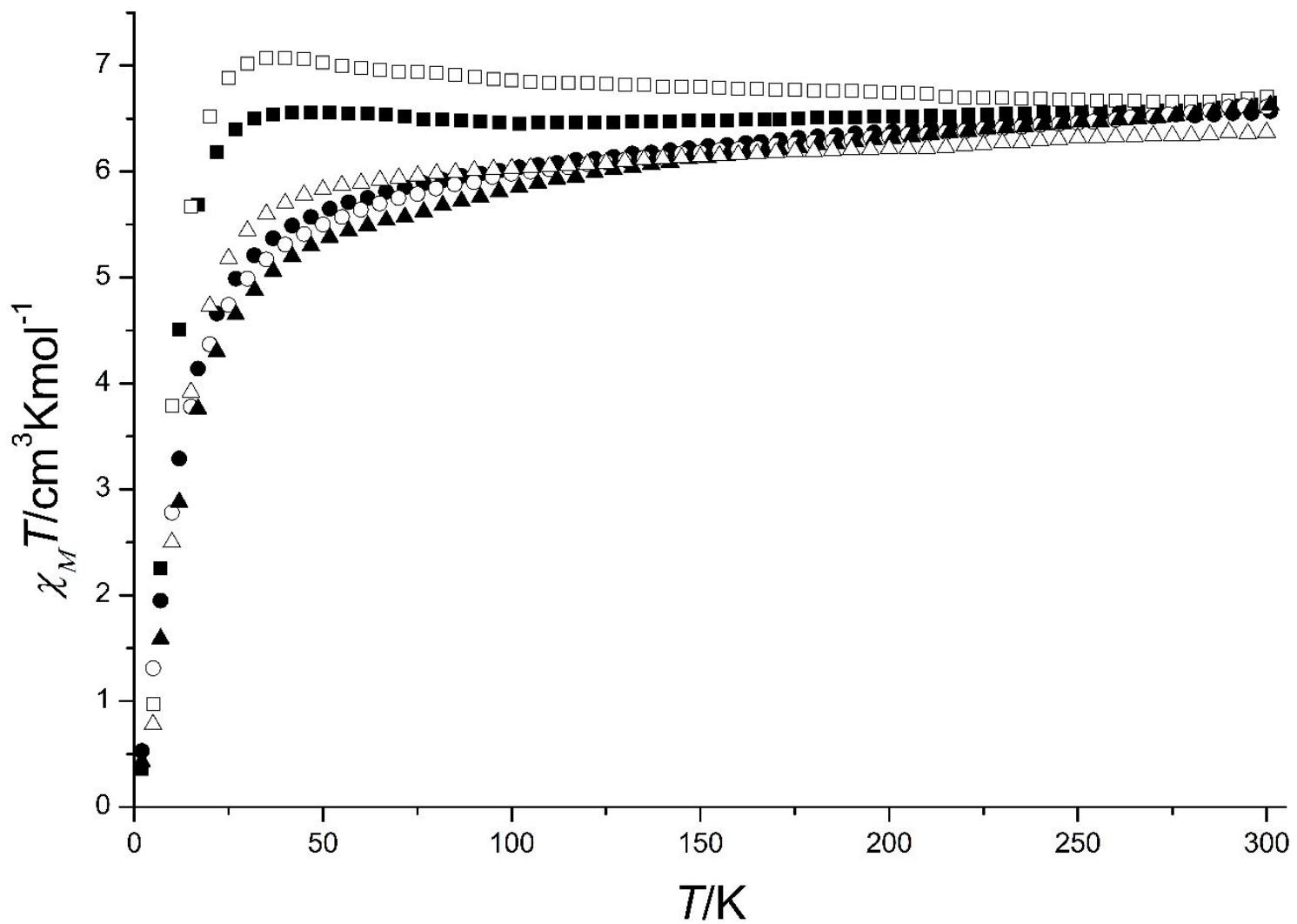

Figure S29: $\chi_{M} T$ vs $T$ data for the compound $\mathbf{C 1}$ (freshly prepared: filled squares; dried: open squares), C2 (freshly prepared: filled triangles; dried: open triangles) and $\mathbf{C} 3$ (freshly prepared: filled dots; dried: open dots). The Data is given per dinuclear iron(II) molecule.

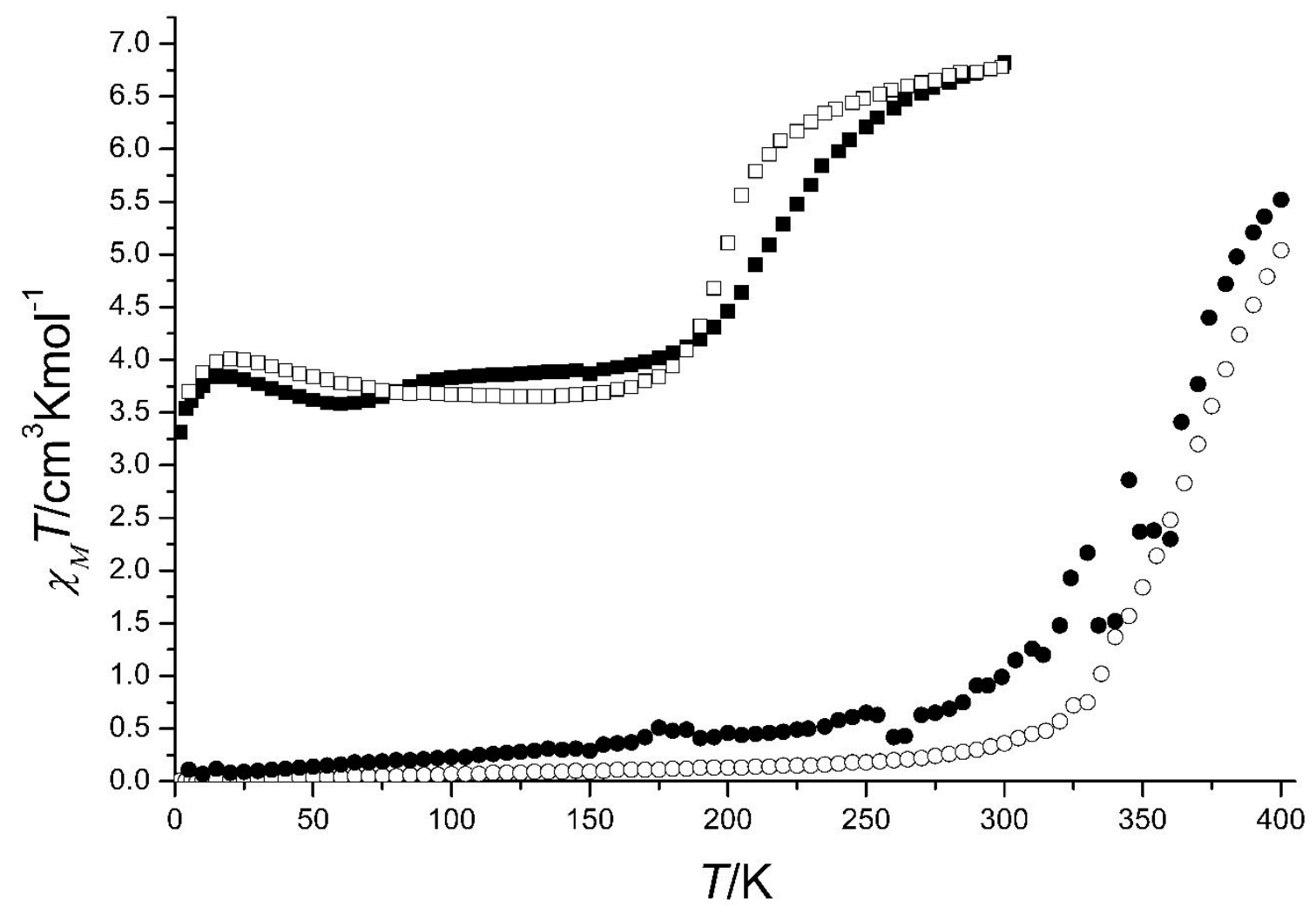

Figure S30: $\chi_{M} T$ vs $T$ data for the compound $\mathbf{C 4}$ (freshly prepared: filled squares; dried: open squares), and C6 (freshly prepared: filled dots; dried: open dots). The Data is given per dinuclear iron(II) molecule. 


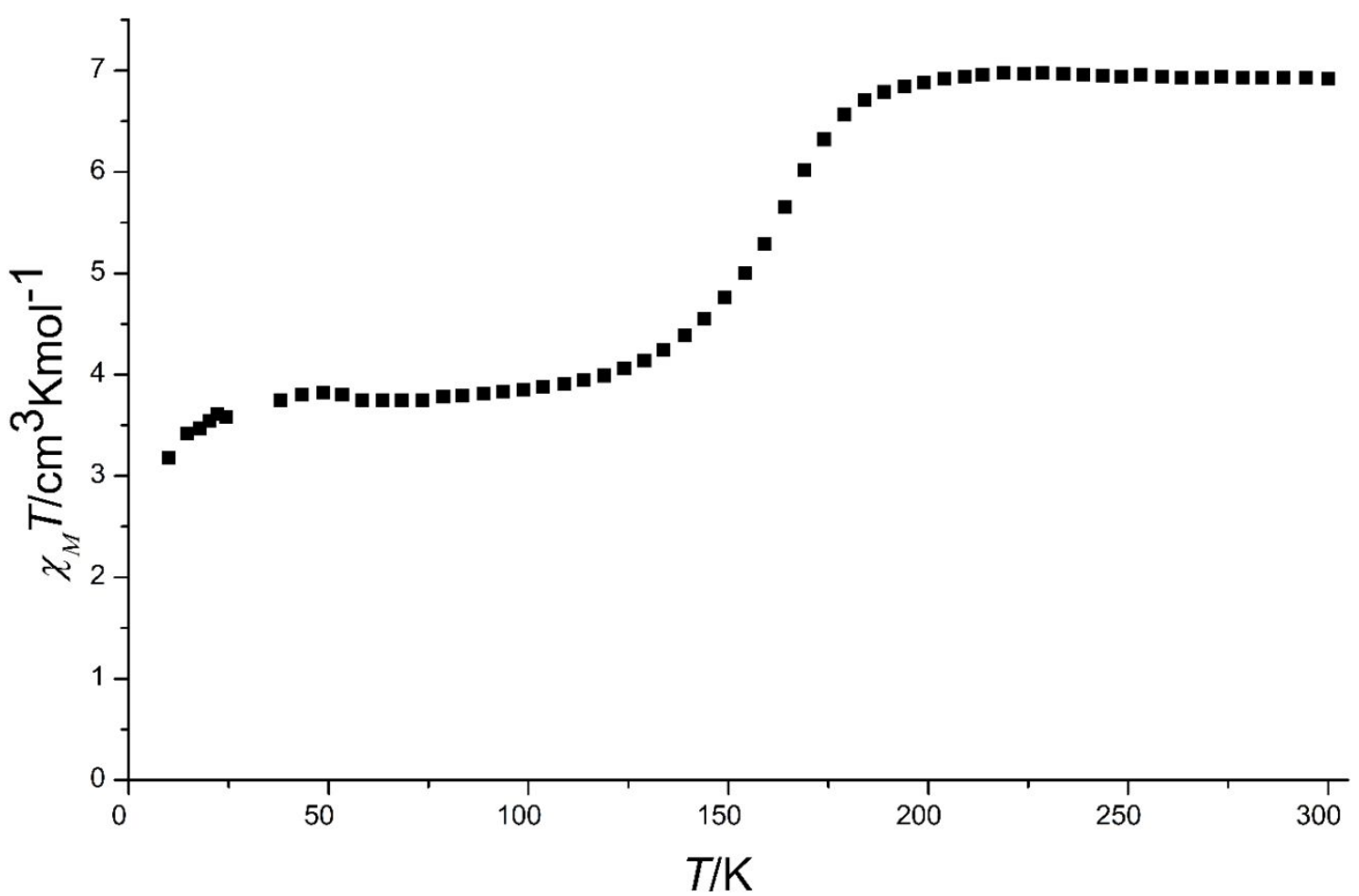

Figure S31: $\chi_{M} T$ vs $T$ data for $\mathbf{C 5}$ in sweeping mode with a cooling rate of $10 \mathrm{~K} / \mathrm{min}$. The Data is given per dinuclear iron(II) molecule. 